\title{
THE EVOLUTION OF ACTIVE GALACTIC NUCLEI IN CLUSTERS OF GALAXIES TO REDSHIFT 1.3
}

\author{
Paul Martini ${ }^{1}$, Gregory R. SivakofF ${ }^{1,3}$, and John S. Mulchaey ${ }^{2}$ \\ ${ }^{1}$ Department of Astronomy and Center for Cosmology and Astroparticle Physics, The Ohio State University, 140 West 18th Avenue, Columbus, OH 43210, USA; \\ martini@astronomy.ohio-state.edu \\ ${ }^{2}$ Carnegie Observatories, 813 Santa Barbara St., Pasadena, CA 91101-1292, USA \\ Received 2009 April 2; accepted 2009 June 8; published 2009 July 20
}

\begin{abstract}
We have measured the luminous active galactic nucleus (AGN) population in a large sample of clusters of galaxies and find evidence for a substantial increase in the cluster AGN population from $z \sim 0.05$ to $z \sim 1.3$. The present sample now includes 32 clusters of galaxies, including 15 clusters above $z=0.4$, which corresponds to a three-fold increase compared to our previous work at high redshift. At $z<0.4$, we have obtained new observations of AGN candidates in six additional clusters and found no new luminous AGN in cluster members. Our total sample of 17 low-redshift clusters contains only two luminous AGNs, while at high redshifts there are 18 such AGNs, or an average of more than one per cluster. We have characterized the evolution of luminous X-ray AGNs as the fraction of galaxies with $M_{R}<M_{R}^{*}(z)+1$ that host AGNs with rest-frame, hard X-ray [2-10 keV] luminosities $L_{X, H} \geqslant 10^{43}$ erg s${ }^{-1}$. The AGN fraction increases from $f_{A}=0.134_{-0.087}^{+0.18} \%$ at a median $z=0.19$ to $f_{A}=1.00_{-0.23}^{+0.29} \%$ at a median $z=0.72$. Our best estimate of the evolution is a factor of 8 increase to $z=1$ and the statistical significance of the increase is $3.8 \sigma$. This dramatic evolution is qualitatively similar to the evolution of the starforming galaxy population in clusters known as the Butcher-Oemler effect. We discuss the implications of this result for the coevolution of black holes and galaxies in clusters, the evolution of AGN feedback, searches for clusters with the Sunyaev-Zel'dovich effect, and the possible detection of environment-dependent downsizing.
\end{abstract}

Key words: galaxies: active - galaxies: clusters: general - galaxies: evolution - X-rays: galaxies - X-rays: galaxies: clusters - X-rays: general

\section{INTRODUCTION}

The demographics of active galactic nuclei (AGNs) in clusters of galaxies have important implications for the growth of the supermassive black holes at the centers of cluster galaxies, the nature of AGN fueling, and the impact of AGNs on the intracluster medium (ICM) over cosmic time. The luminous, massive elliptical galaxies that dominate the galaxy population in the richest clusters are also expected (and in some cases are measured; Houghton et al. 2006; Gebhardt et al. 2007) to have the most massive black holes in the local universe. As the stars in these galaxies appear to have an earlier mean formation epoch than those in field galaxies (e.g., van Dokkum \& Franx 1996; Kelson et al. 1997), the apparent coevolution of black holes and galaxies (e.g., Hopkins et al. 2006, and references therein) implies that the bulk of their present black hole mass was also accreted at earlier times.

This scenario is also motivated by observations of local clusters that clearly show their galaxy populations are more quiescent than local field galaxies. An early demonstration by Osterbrock (1960) showed that cluster ellipticals were far less likely to have [O II] $\lambda 3727$ emission than field ellipticals, a result that has since been confirmed by many studies (e.g., Gisler 1978; Dressler et al. 1985, 1999). One big question that has motivated this work is: why are galaxy populations different in clusters? Numerous physical mechanisms have been invoked to explain the relative lack of star formation in cluster galaxies, as well as their higher fraction of elliptical and S0 galaxies (Dressler 1980) and relative lack of cold gas (e.g., Giovanelli \& Haynes 1985). These include ram-pressure stripping by the ICM (Gunn \& Gott 1972), evaporation of a galaxy's interstellar

\footnotetext{
3 Current Address: Department of Astronomy, University of Virginia, P.O. Box 400325, Charlottesville, VA 22904-4325.
}

medium (ISM) by the hot ICM (Cowie \& Songaila 1977), tidal effects with the cluster potential (Farouki \& Shapiro 1981; Merritt 1983; Byrd \& Valtonen 1990), the absence of newly accreted cold gas (Larson et al. 1980), and galaxy harassment and mergers (Richstone 1976; Moore et al. 1996).

All of these physical effects may also be important for fueling accretion onto the central black holes in galaxies because they impact either the available gas supply in a galaxy, angular momentum transport, or both. The best and perhaps only candidate process for fueling the most luminous AGNs is the merger of two gas-rich galaxies (e.g. Barnes \& Hernquist 1992) and the relative lack of both cold gas and major mergers is a reasonable explanation for the nearly complete absence of QSOs hosted by cluster galaxies. For less luminous AGNs, the case is less clear because an increasing number of physical processes such as minor mergers, galaxy harassment, various types of bars, stellar mass loss, etc. could also play a role (see Martini 2004 , for a review). If mechanisms such as galaxy harassment and stellar mass loss are important for fueling low-luminosity AGNs, then comparable numbers of low-luminosity AGNs may be present in clusters and the field.

Recent studies of the AGN fraction as a function of environment with emission-line galaxies from the Sloan Digital Sky Survey (SDSS) find that the most luminous AGNs are rarer in denser environments (SDSS; Kauffmann et al. 2004; Popesso \& Biviano 2006), although these studies do not sample the densest regions of clusters well. This decrease is in contrast to both lower-luminosity AGNs in SDSS (Miller et al. 2003) and radio observations (Best 2004; Best et al. 2005), which show that the radio AGN fraction does not decrease significantly in denser environments. X-ray observations with Chandra show that the X-ray AGN fraction is larger than expected from AGN selection via visible-wavelength emission lines. In previous 
work, we showed that X-ray observations identified approximately five times as many AGNs as selection at visiblewavelengths (Martini et al. 2002, 2006), although the precise value of the X-ray excess depends significantly on the relative sensitivity and luminosity threshold of the observations. This spectroscopic study of X-ray counterparts confirmed the many previous studies that suggested a higher X-ray AGN population in clusters from surface density arguments alone (e.g. Cappi et al. 2001; Sun \& Murray 2002; Ruderman \& Ebeling 2005), yet it is not clear if the X-ray AGN fraction is higher than the field value. To date there is only weak evidence that the $\mathrm{X}$ ray AGN fraction in clusters is comparable to the fraction in field early-type galaxies (Lehmer et al. 2007; Sivakoff et al. 2008; Arnold et al. 2009). One of the virtues of the emissionline galaxy studies as a function of environment is that they can directly calculate the fraction of a given galaxy population that hosts AGNs as a function of environment, even though this technique appears to systematically miss AGNs in the densest regions relative to $\mathrm{X}$-ray and radio selection.

In addition to a local comparison between AGNs in different environments, measurement of the evolution of the AGN population in clusters can constrain the formation epoch for their supermassive black holes and the extent of their coevolution with the cluster galaxy population. The key early work on the evolution of galaxies in clusters was by Butcher \& Oemler (1978, 1984), who observed a substantial increase in the fraction of blue galaxies in higher-redshift clusters. The Butcher-Oemler effect is interpreted as an increase in the amount of star formation and has been confirmed by many other indicators, in particular [O II] emission-line galaxy fractions (Poggianti et al. 2006) and an increase in the number of $24 \mu \mathrm{m}$ sources in Spitzer observations of distant clusters (Bai et al. 2007; Saintonge et al. 2008). The observed increase brings the star formation rate (SFR) in cluster galaxies closer to those in the field. At a redshift of $z \sim 1$ and higher, observations with Spitzer even find that galaxies in denser environments have higher SFRs than lower-density regions (Elbaz et al. 2007), which is opposite the trend observed in the local universe. Similar results have also been found with deep UV data (Heinis et al. 2007). The situation is less clear when star formation is measured with the [O II] emission line because while Poggianti et al. (2008) found that star formation does not strongly depend on environment, Cooper et al. (2008) found the specific SFR has a similar dependence on environment at $z=0$ and $z=1$, although the total star formation rate is higher in clusters at $z=1$ than in the field.

The existence of the Butcher-Oemler effect and the many indirect arguments outlined above for a connection between star formation and black hole accretion suggest that there should be an increased AGN population in high-redshift clusters. An early study of the high-redshift cluster 3C295 at $z=0.46$ by Dressler \& Gunn (1983) found evidence for three AGNs and was an indication that this may be the case; however, their relative scarcity precluded a detailed statistical study or targeted studies to deliberately identify cluster AGNs. This situation changed dramatically with the launch of Chandra, whose superb sensitivity and angular resolution produced a dramatic increase in efficiency for searches for AGNs, particularly lower-luminosity sources. Just as the case for local clusters, Chandra observations of distant clusters have revealed substantial populations of point sources (Cappelluti et al. 2005; Gilmour et al. 2009). Spectroscopic confirmation that these point sources are associated with cluster members has been more challenging (Johnson et al. 2003; Demarco et al. 2005), but in Eastman et al. (2007) we combined new observations of MS2053.7-0449 $(z=0.58)$ with archival data on three additional, $z>0.5$ clusters and found an approximate order of magnitude increase in the fraction of $M_{R}<-20$ mag galaxies that hosted AGNs more luminous than $L_{X, H} \geqslant 10^{43} \mathrm{erg} \mathrm{s}^{-1}$ in the hard X-ray band (2$10 \mathrm{keV}$ ) relative to the sample of 10 low-redshift $z<0.32$ clusters in Martini et al. (2007). These results have since been strengthened with detailed studies of clusters at $z \sim 1$ with XMM-Newton (van Breukelen et al. 2009) and measurements of surface density excesses in clusters to $z \sim 1.5$ (Galametz et al. 2009).

In addition to their application to the coevolution of black holes and galaxies, an increase in the AGN fraction in clusters may also impact the ICM. At low redshifts many studies have shown that AGN feedback is a viable explanation for the absence of substantial reservoirs of cold gas at the centers of clusters (for a recent review see McNamara \& Nulsen 2007). This feedback is ascribed to AGNs associated with the central cluster galaxy, which is almost invariably a luminous radio source. In our studies of X-ray AGNs, this is almost the only cluster galaxy in which we are insensitive to the presence of an AGN because it is challenging to measure even a bright nuclear point source when juxtaposed with the extended emission from the ICM that often peaks near the central cluster galaxy. Nevertheless, the evolution of AGNs in other cluster galaxies is likely to be connected to the evolution of the central AGN as the stars in the most luminous cluster galaxies have comparable ages. An increase in the net energy production by AGNs in higher-redshift clusters is of interest because energy input during cluster formation has been invoked as an explanation for the minimum entropy level in the ICM (Kaiser 1991; Evrard \& Henry 1991). AGNs remain perhaps the most viable mechanism, if only because most others can be ruled out (Kravtsov et al. 2004; Conroy \& Ostriker 2008), although the details of how AGN feedback couples to the ICM remain uncertain. Outside of the central galaxy, an increase in the number of other AGNs associated with clusters of galaxies may also affect measurement of other cluster properties (Branchesi et al. 2007; Bignamini et al. 2008). Finally, an analogous increase in the radio-loud AGN population in high-redshift clusters may contaminate searches for clusters via the Sunyaev-Zel'dovich effect (Sunyaev \& Zeldovich 1970) at $\mathrm{mm}$ and $\mathrm{cm}$ wavelengths. As many searches for clusters that exploit this effect are in progress, it is important to characterize the potential impact of evolution of the cluster AGN population on these experiments (e.g., Lin \& Mohr 2007).

In the next section, we describe our expanded high-redshift data, as well as the selection criteria for X-ray AGNs we employ at all redshifts. We then describe our new observations of low-redshift clusters in Section 3. These two data sets are combined to calculate the cluster AGN fraction and its evolution in Section 4, followed by an examination of the properties of the cluster AGNs in Section 5. We discuss the implications of these results, particularly on the coevolution of black holes and galaxies, in Section 6 and conclude with a summary of our results. Throughout this paper we assume that the cosmological parameters are: $\left(\Omega_{M}, \Omega_{\Lambda}, h\right)=(0.3,0.7$, $0.7)$ where $H_{0}=100 \mathrm{hm} \mathrm{s}^{-1} \mathrm{Mpc}^{-1}$. All absolute magnitudes quoted in this paper assume $h=0.7$.

\section{HIGH-REDSHIFT DATA}

Two large surveys have obtained redshifts for substantial numbers of galaxies with X-ray counterparts in many deep, 
Table 1

High-Redshift Cluster Sample

\begin{tabular}{|c|c|c|c|c|c|c|c|c|c|}
\hline $\begin{array}{c}\text { Cluster } \\
\text { (1) }\end{array}$ & $\begin{array}{l}\alpha_{c} \\
(2)\end{array}$ & $\begin{array}{l}\delta_{c} \\
(3)\end{array}$ & $\begin{array}{c}z \\
(4)\end{array}$ & $\begin{array}{c}\sigma\left(\mathrm{km} \mathrm{s}^{-1}\right) \\
(5)\end{array}$ & $\begin{array}{c}\sigma \text { Ref } \\
(6)\end{array}$ & $\begin{array}{c}T_{X}(\mathrm{keV}) \\
(7)\end{array}$ & $\begin{array}{c}T_{X} \operatorname{Ref} \\
(8)\end{array}$ & $\begin{array}{c}R_{200}(\mathrm{Mpc}) \\
(9)\end{array}$ & $\begin{array}{c}\text { Spectra } \\
(10)\end{array}$ \\
\hline MS $1621.5+2640$ & $16: 23: 34.9$ & $+26: 34: 21$ & 0.43 & 735 & 1 & 7.6 & 1 & 1.42 & SESXI \\
\hline $3 C 295$ & $14: 11: 20.5$ & $+52: 12: 09$ & 0.46 & 1642 & 1 & 5.3 & 1 & 3.12 & SESXI \\
\hline MS 0451.6-0305 & $4: 54: 11.1$ & $-03: 00: 55$ & 0.538 & 1371 & 2 & 8.1 & 1 & 2.49 & ChaMP \\
\hline MS 0015.9+1609 & $0: 18: 33.5$ & $+16: 26: 06$ & 0.541 & 1234 & 2 & 9.4 & 2 & 2.24 & ChaMP \\
\hline RX J0848.7+4456 & $8: 48: 47.6$ & $+44: 56: 16$ & 0.574 & 670 & 3 & 3.2 & 3 & 1.19 & SESXI \\
\hline MS 2053.7-0449 & $20: 56: 21.3$ & $-04: 37: 49$ & 0.583 & 865 & 4 & 5.2 & 1 & 1.53 & SESXI,ChaMP \\
\hline RX J0542.8-4100 & $5: 42: 49.8$ & $-41: 00: 07$ & 0.634 & 1101 & 3 & 7.9 & 3 & 1.89 & ChaMP \\
\hline RX J2302.8+0844 & $23: 02: 48.3$ & $+08: 43: 48$ & 0.722 & 993 & 3 & 6.6 & 3 & 1.61 & ChaMP \\
\hline MS $1137.5+6625$ & 11:40:22.1 & $+66: 08: 14$ & 0.782 & 967 & 3 & 6.3 & 1 & 1.52 & ChaMP \\
\hline RX J1317.4+2911 & $13: 17: 22.0$ & $+29: 11: 24$ & 0.805 & 531 & 3 & 2.2 & 1 & 0.82 & SESXI \\
\hline RX J1716.4+6708 & $17: 16: 49.3$ & $+67: 08: 25$ & 0.813 & 1445 & 1 & 6.6 & 1 & 2.22 & SESXI,ChaMP \\
\hline MS 1054-03 & $10: 56: 55.7$ & $-03: 37: 39$ & 0.831 & 1156 & 5 & 7.8 & 1 & 1.76 & ChaMP \\
\hline RDCS J0910+5422 & $9: 10: 44.7$ & $+54: 22: 04$ & 1.11 & 675 & 6 & 3.5 & 1 & 0.87 & SESXI \\
\hline
\end{tabular}

Notes. Cluster sample and properties derived from the present study. Columns are: (1) Cluster name; (2 and 3$)$ R.A. and decl. for the centroid of the extended X-ray emission; (4) redshift; (5) velocity dispersion; (6) reference for the velocity dispersion; (7) X-ray temperature in keV; (8) reference for the X-ray temperature; (9) estimate of the virial radius in Mpc (e.g., Treu et al. 2003); (10) origin of most of the spectra. References for velocity dispersion are: 1: Girardi \& Mezzetti (2001); 2: Carlberg et al. (1996); 3: derived from the X-ray temperature following Xue \& Wu (2000); 4: Tran et al. (2005); 5: Tran et al. (2007); 6: Mei et al. (2006); 7: from weak lensing estimate Jee et al. (2006); 8: Stanford et al. (2001). References for X-ray temperatures are: 1: Vikhlinin et al. (2002); 2: Ebeling et al. (2007); 3: Ettori et al. (2004); 4: Jee et al. (2006).

archival Chandra observations that include substantial numbers of high-redshift clusters of galaxies. These are the Serendipitous Extragalactic X-ray Source Identification Program (SEXSI; Harrison et al. 2003; Eckart et al. 2005, 2006) and the Chandra Multiwavelength Project (ChaMP; Kim et al. 2004a, 2004b; Green et al. 2004; Silverman et al. 2005). We have investigated the fields surveyed by both SEXSI and ChaMP to identify data sets that contain clusters of galaxies with $z>0.4$ and have sufficient depth to identify $L_{X, H} \geqslant 10^{43} \mathrm{erg} \mathrm{s}^{-1}$ (rest frame 2-10 keV) AGNs at the cluster redshift.

The SEXSI survey published spectroscopic redshifts for 27 archival Chandra observations in Eckart et al. (2006) that were selected to identify hard X-ray sources over the flux range of $10^{-13}-10^{-15} \mathrm{erg} \mathrm{s}^{-1} \mathrm{~cm}^{-2}$ and isolate those responsible for the hard X-ray background. The specific selection criteria for the fields were that they must be high Galactic latitude $\left(|b|>20^{\circ}\right)$ and be obtained with either the I or S modes of the Advanced Camera for Imaging Spectroscopy (ACIS; Bautz et al. 1998) when no grating was used. The X-ray luminosities quoted by SEXSI are based on spectral fits that assume a $\Gamma=1.5$ power law and intrinsic absorption $N_{H}$ at the source redshift, although they quote the observed luminosities (not corrected for obscuration) and provide the best-fit $N_{H}$ value. The average spectroscopic completeness is $67 \%$ (see Section 4.2 below) for sources with $R<24.4 \mathrm{mag}$ on the Vega system. Nine of the 27 SEXSI fields include clusters of galaxies with $z>0.4$ and we include seven ${ }^{4}$ in our sample. As one field contains three clusters, we list nine clusters from SEXSI in Table 1.

The ChaMP survey published spectroscopic redshifts for 20 archival Chandra observations in Silverman et al. (2005) that were similarly selected for depth, high Galactic latitude $\left(|b|>20^{\circ}\right)$, and no special observing modes. The spectroscopic

4 RX J1350.0+6007 was not targeted for spectroscopy and the X-ray data for CL0442+0202 $(z=1.11)$ were sufficiently shallow $(t=44 \mathrm{ks})$ that they may not be complete to $L_{X, H}=10^{43} \mathrm{erg} \mathrm{s}^{-1}$. In addition, Stern et al. (2003) classify CL0442+0202 as an overdensity that has not yet collapsed, rather than as a cluster. completeness of ChaMP is 77\% at $r^{\prime}<22.5 \mathrm{mag}$, where $r^{\prime}$ is on the SDSS photometric system (Fukugita et al. 1996, and $\left.r_{A B}^{\prime}=R_{\text {Vega }}+0.17\right)$. Their X-ray luminosities are based on spectral fits that assume a $\Gamma=1.9$ power law and intrinsic absorption $N_{H}$ at the source redshift, as well as the appropriate Galactic absorption, although they also quote the observed luminosities (only corrected for Galactic absorption). The final sample presented in Silverman et al. (2005) was restricted to X-ray sources with $L_{X}>10^{42} \mathrm{keV}$ in the $2-8 \mathrm{keV}$ band in order to ensure all are AGNs. Most (69\%) are spectroscopically classified as broad-line AGN (BLAGN). 9 of these 20 ChaMP fields include clusters of galaxies with $z>0.4$ and we include eight ${ }^{5}$ of these in our study (see Table 1). Two of these clusters are common to both ChaMP and SEXSI (MS2053.7-0449 and RXJ1716.4+6708) and therefore the final sample has 15 clusters with $z>0.4$. While spectroscopic data for X-ray sources in other high-redshift clusters exist (e.g. Johnson et al. 2006), we limit our high-redshift sample to these 15 to maximize the uniformity of the data set.

We have also compiled additional data for each cluster listed in Table 1 that will be important for our subsequent analysis. One quantity is the center of the cluster, which is needed to determine if a given AGN falls within the projected virial radius of the cluster. We associate the center of each cluster with the centroid of the extended X-ray emission. While these coordinates do not always agree with the standard coordinates quoted in the literature, this assumption makes our analysis more uniform. The redshift and velocity dispersion are also needed to determine if an AGN is within the cluster. In most cases, velocity dispersions for these clusters are available in the literature and we quote the origin of the measurement we adopt in the table. When the velocity dispersion has not been measured, we estimate this quantity from the X-ray temperature and the $\sigma-T_{X}$ relationship from Xue \& Wu (2000). Specifically,

\footnotetext{
5 We exclude CL J0152.7-1357 $(z=0.831)$ because the exposure time is shorter than the others at $t=34.6 \mathrm{ks}$ and therefore the X-ray data may not be complete to $L_{X, H}=10^{43} \mathrm{erg} \mathrm{s}^{-1}$.
} 
Table 2

High-Redshift Cluster AGN Sample

\begin{tabular}{|c|c|c|c|c|c|c|c|c|}
\hline $\begin{array}{l}\mathrm{AGN} \\
(1)\end{array}$ & $\begin{array}{l}\text { Cluster } \\
(2)\end{array}$ & $\begin{array}{c}z \\
(3)\end{array}$ & $\begin{array}{c}R(\mathrm{mag}) \\
(4)\end{array}$ & $\begin{array}{c}\log L_{X, H}\left(\mathrm{erg} \mathrm{s}^{-1}\right) \\
(5)\end{array}$ & $\begin{array}{c}\delta v / \sigma \\
(6)\end{array}$ & $\begin{array}{c}\Delta R(\operatorname{arcmin}) \\
(7)\end{array}$ & $\begin{array}{l}R / R_{200} \\
\quad(8)\end{array}$ & $\begin{array}{l}\text { Class } \\
(9)\end{array}$ \\
\hline CXOSEXSI J141127.4+521131 & $3 \mathrm{C} 295$ & 0.451 & 19.78 & 43.4 & 1.13 & 1.23 & 0.14 & ALG \\
\hline CXOSEXSI J141123.4+521331 & $3 \mathrm{C} 295$ & 0.472 & 19.05 & 43.8 & 1.5 & 1.45 & 0.16 & BLAGN \\
\hline E0015+162 & MS0015.9+1609 & 0.553 & 18.41 & 45.48 & 1.89 & 3.35 & 0.58 & BLAGN \\
\hline CXOSEXSI J084858.0+445434 & RX J0848.7+4456 & 0.573 & 19.58 & 43.8 & 0.28 & 2.5 & 0.83 & BLAGN \\
\hline CXOMP J054248.2-410140 & RDCSJ0542-4100 & 0.634 & 20.64 & 43.24 & 0 & 1.58 & 0.32 & NELG \\
\hline CXOMP J054251.4-410205 & RDCSJ0542-4100 & 0.637 & 19.63 & 43.35 & 0.5 & 1.99 & 0.33 & ALG \\
\hline CXOMP J054259.5-410241 & RDCSJ0542-4100 & 0.638 & 20.50 & 43.37 & 0.67 & 3.16 & 0.63 & NELG \\
\hline CXOMP J054240.8-405626 & RDCSJ0542-4100 & 0.639 & 20.89 & 43.67 & 0.83 & 4.05 & 0.81 & NELG \\
\hline CXOMP J054255.0-405922 & RDCSJ0542-4100 & 0.644 & 22.08 & 43.08 & 1.67 & 1.24 & 0.25 & NELG \\
\hline CXOMP J114022.0+660816 & MS1137+6625 & 0.786 & 20.37 & 43.24 & 0.7 & 0.04 & 0.01 & BLAGN \\
\hline CXOSEXSI J171636.9+670829 & RXJ1716.4+6708 & 0.795 & 22 & 44 & 2.06 & 1.19 & 0.24 & ELG \\
\hline CXOSEXSI J131718.8+291111 & RX J1317.4+2911 & 0.803 & 21.98 & 43.3 & 0.63 & 0.68 & 0.38 & BLAGN \\
\hline CXOSEXSI J171703.8+670900 & RXJ1716.4+6708 & 0.812 & 21.79 & 43 & 0.11 & 1.53 & 0.31 & ELG \\
\hline CXOSEXSI J171714.5+671136 & RXJ1716.4+6708 & 0.815 & 22.68 & 43.2 & 0.23 & 4.02 & 0.82 & ELG \\
\hline CXOMP J105650.6-033508 & MS 1054-03 & 0.818 & 21.76 & 43.22 & 1.84 & 2.82 & 0.73 & BLAGN \\
\hline CXOU J091043.3+542152 & RDCSJ0910+5422 & 1.104 & 24 & 43.06 & 1.26 & 0.29 & 0.16 & AGN2 \\
\hline CXOSEXSI J084905.3+445203 & LynxE & 1.266 & 24.61 & 43.8 & 1.11 & 1.27 & 0.74 & ELG \\
\hline CXOSEXSI J084831.6+445442 & LynxW & 1.267 & 25.42 & 43.2 & 0.61 & 1.23 & 0.8 & ELG \\
\hline
\end{tabular}

Notes. AGNs in high-redshift clusters of galaxies. Columns are: (1) AGN name; (2) Cluster; (3) AGN redshift; (4) $R$-band magnitude; (5) Rest-frame, hard-X-ray luminosity (2-10 keV); (6) Velocity offset from the cluster systemic velocity normalized by the cluster velocity dispersion; (7) Projected radial offset relative to the centroid of the X-ray gas in arcminutes; (8) Projected radial offset normalized by the cluster virial radius; (9) Spectroscopic classification. The $R$-band magnitude of E0015+162 is from Örndahl et al. (2003). The remaining values are from either Eckart et al. (2006) for the SEXSI sample or from Silverman et al. (2005) for the ChaMP sample (although corrected from $r^{\prime}$ to $R$ as noted in Section 2). The 2-8 keV X-ray luminosities from Silverman et al. (2005) have been corrected to the $2-10 \mathrm{keV}$ band as described in Section 2.

we used the relation $\sigma=10^{2.51 \pm 0.01} T^{0.61 \pm 0.01} \mathrm{~km} \mathrm{~s}^{-1}$ derived from their combined group and cluster sample with orthogonal distance regression (Feigelson \& Babu 1992). Based on their data, we estimate that there is a $30 \%$ uncertainty in $\sigma$ at fixed $T$.

One potential concern for our subsequent analysis is that the Xue \& Wu (2000) $\sigma-T$ relation may not hold at higher redshift. Lubin et al. (2004) investigated this point for several optically selected clusters and found that they were 2-9 times cooler than expected from the local relation; however, the difference was much less stark for X-ray selected, high-redshift clusters similar (and in several cases identical to) those presented here. Fang et al. (2007) showed that high-redshift, X-ray-selected clusters are consistent with the low-redshift $L_{X}-\sigma$ relation, although spectroscopically selected groups and clusters do not agree as well (see also Andreon et al. 2008).

Finally, we have calculated the projected size of the virial radius for each cluster following Treu et al. (2003) and throughout this paper we associate the virial radius with $R_{200}$, the radius within which the cluster is a factor of 200 overdensity. Of the three clusters we have in common with Poggianti et al. (2006), for 3C 295 and MS1054-03 we adopt nearly the same $\sigma$ and our $R_{200}$ estimate is nearly identical to theirs, while for MS0015.9+1609 we adopt a slightly larger velocity dispersion (1234 $\mathrm{km} \mathrm{s}^{-1}$ from Carlberg et al. (1996) rather than their $984 \mathrm{~km} \mathrm{~s}^{-1}$ ) and consequently infer a larger radius.

Because the most relevant ChaMP measurements are the 2$8 \mathrm{keV}$ luminosity, rather than 2-10 keV luminosity, we multiply the ChaMP 2-8 keV luminosities by a factor of 1.2. This correction factor was calculated for a $\Gamma=1.7$ power law with PIMMS. There is some uncertainty in this correction factor because not all AGNs have this power-law form, particularly as we assume this correction for their observed rather than intrinsic (unobscured) spectra, but this is not a significant effect compared to other sources of systematic errors that we discuss below. There are no additional AGNs from ChaMP that enter the sample after this step because there are none just below the $10^{43} \mathrm{erg} \mathrm{s}^{-1}$ threshold in the $2-8 \mathrm{keV}$ band. We also estimated the difference in luminosity for an AGN calculated with the $\Gamma=1.5$ power law employed by SEXSI, the $\Gamma=1.9$ employed by ChaMP, and a $\Gamma=1.7$ power law to determine if these differences would cause any sources to fall in or out of the same and none would do so. In the two clusters observed by both ChaMP and SEXSI, there is one cluster AGN common to both surveys: CXOSEXSI J171636.9+670829. The redshifts from the two surveys agree exactly $(z=0.795)$ and the luminosities agree well: $L_{X, 2-10}=10^{44} \mathrm{erg} \mathrm{s}^{-1}$ and $L_{X, 2-8}=10^{43.88} \mathrm{erg} \mathrm{s}^{-1}$.

We also correct the ChaMP $r^{\prime}$ measurements to the Vega $R$ band as discussed above. Based on the magnitudes of these sources and a simple $k$-correction, we estimate that none of these sources falls below our galaxy luminosity threshold. As these are fairly luminous AGNs, in some cases the AGN may dominate the total flux and we may have overestimated the host galaxy luminosity. E0015+162 (Margon et al. 1983) is the most X-ray luminous AGN in our sample by over an order of magnitude and is a useful case study to test the importance of this concern. This AGN has a total $R=18.41 \mathrm{mag}$ and a fainter host galaxy magnitude of $R=19.8 \mathrm{mag}$ (Örndahl et al. 2003), which corresponds to a factor of 3.6 in flux. If the other AGNs have similar or smaller $L_{R} / L_{X}$ ratios (such as due to obscuration), then we expect their AGN contribution to the measured $R$-band flux to be negligible because they are all much less luminous than E0015+162.

We identify AGNs in these clusters with the following four criteria: (1) the hard X-ray luminosity must be $L_{X, H} \geqslant 10^{43}$ $\mathrm{erg} \mathrm{s}^{-1}$; (2) the AGN redshift must fall within $3 \sigma$ of the cluster mean redshift, where $\sigma$ is the cluster velocity dispersion; (3) the AGN must fall within the projected virial radius $R_{200}$ of 
Table 3

New Low-Redshift Clusters

\begin{tabular}{lcccccccc}
\hline \hline \multicolumn{1}{c}{\begin{tabular}{c} 
Cluster \\
\multicolumn{1}{c}{$(1)$}
\end{tabular}} & $\begin{array}{c}\alpha_{c} \\
(2)\end{array}$ & $\begin{array}{c}\delta_{c} \\
(3)\end{array}$ & $\begin{array}{c}z \\
(4)\end{array}$ & $\begin{array}{c}\sigma\left(\mathrm{km} \mathrm{s}^{-1}\right) \\
(5)\end{array}$ & $\begin{array}{c}\sigma \operatorname{Ref} \\
(6)\end{array}$ & $\begin{array}{c}T_{X}(\mathrm{keV}) \\
(7)\end{array}$ & $\begin{array}{c}T_{X} \operatorname{Ref} \\
(8)\end{array}$ & $\begin{array}{c}R_{200}(\mathrm{Mpc}) \\
(9)\end{array}$ \\
\hline Abell 1240 & $11: 23: 37.3$ & $+43: 06: 54$ & 0.1590 & 698 & 1 & $\ldots$ & $\ldots$ & 1.64 \\
Abell 1942 & $14: 38: 22.0$ & $+03: 40: 07$ & 0.2240 & 903 & 2 & 5.6 & 1 & 1.96 \\
Abell 2125 & $15: 41: 13.2$ & $+66: 16: 01$ & 0.2465 & 1113 & 3 & 3.2 & 2 & 2.39 \\
MS1455.0+2232 & $14: 57: 15.1$ & $+22: 20: 29$ & 0.2578 & 1032 & 4 & 5.5 & 3 & 2.20 \\
ZwCl 1358.1+6245 & $13: 59: 50.6$ & $+62: 31: 04$ & 0.3280 & 1003 & 4 & 6.5 & 3 & 2.06 \\
MS1512.4+3647 & $15: 14: 22.4$ & $+36: 36: 21$ & 0.3720 & 575 & 4 & 3.6 & 3 & 1.15 \\
\hline
\end{tabular}

Notes. New low-redshift clusters and their properties derived from the present study. Columns are: (1) Cluster name; (2 and 3) R.A. and decl. for the centroid of the extended X-ray emission; (4) redshift; (5) velocity dispersion; (6) reference for the velocity dispersion; (7) X-ray temperature in $\mathrm{keV}$; (8) reference for the X-ray temperature; (9) estimate of the virial radius in Mpc (Treu et al. 2003). References for velocity dispersion are: 1: derived from the X-ray luminosity following Xue \& Wu (2000); 2: derived from the X-ray temperature following Xue \& Wu (2000); 3: Miller et al. (2004); 4: Borgani et al. (1999). References for X-ray temperatures are: 1: Ota \& Mitsuda (2004); 2: Wang et al. (2004); 3: Mushotzky \& Scharf (1997).

the cluster; (4) the absolute magnitude of the host galaxy must be greater than $M_{R}=M_{R}^{*}(z)+1$ mag. Most of these criteria were adopted from Eastman et al. (2007), although the absolute magnitude criterion is different and we discuss our motivation for this choice in Section 4.3 below. With these criteria we identify 18 AGNs in the 15 clusters with $z>0.4$, or an average of more than one per cluster. The properties of the $z>0.4$ AGN are presented in Table 2.

\section{NEW LOW-REDSHIFT OBSERVATIONS}

AGNs more luminous than $L_{X, H}=10^{43} \mathrm{erg} \mathrm{s}^{-1}$ are sufficiently rare in low-redshift clusters that Poisson uncertainties (as opposed to sources of systematic errors) from the lowredshift sample may dominate the statistical significance of any evidence of evolution. Our previous study of 10 clusters with $z<0.32$ only identified one AGN above this luminosity threshold (Martini et al. 2006), while our more recent observations of three additional clusters (all at $z<0.08$ ) have identified only one additional AGNs above this luminosity (Sivakoff et al. 2008). We have, therefore, studied six additional clusters with $0.15<z<0.4$ to find other X-ray AGNs more luminous than $L_{X, H}=10^{43} \mathrm{erg} \mathrm{s}^{-1}$ with a combination of Chandra archival data and follow-up spectroscopy of candidate cluster $\mathrm{X}$-ray AGN at the MDM Observatory. These clusters were selected to be the nearest massive clusters in the Chandra archive whose estimated virial radii fit within the Chandra ACIS field of view (FOV) and were accessible during our observing runs. The new clusters and their physical properties are listed in Table 3.

\subsection{Chandra X-ray Analysis}

The X-ray observations were processed following the same techniques employed by Sivakoff et al. (2008). We reduced all data using CIAO $3.4^{6}$ with CALDB 3.3.O.I and NASA's FTOOLs 6. . $^{7}$ The observations are summarized in Table 4. Only minor differences in reduction were required for these archival observations. The majority of the clusters had data with an aimpoint centered on the four ACIS-I chips $\left(\sim 17^{\prime}\right.$ FOV $)$ and frame times of $3.1 \mathrm{~s}$. These data were telemetered and cleaned in Very Faint mode. The more distant clusters, $\mathrm{ZwCl} 1358.1+6245$ and MS 1512.4+3647, were observed with the aimpoint placed on the ACIS-S3 detector (8.4 FOV) and had frame times of $3.3 \mathrm{~s}$.

\footnotetext{
6 http://asc.harvard.edu/ciao/

7 http://heasarc.gsfc.nasa.gov/docs/software/lheasoft/
}

Table 4

Chandra Observation Logs

\begin{tabular}{lcccc}
\hline \hline \multicolumn{1}{c}{ Cluster } & OBSID & Detector & $\begin{array}{c}\mathrm{T} \\
(\mathrm{ks})\end{array}$ & $\begin{array}{c}L_{X, H, \text { Lim }} \\
\left(10^{41} \mathrm{erg} \mathrm{s}^{-1}\right) \\
(1)\end{array}$ \\
\hline Abell 1240 & $(2)$ & $(3)$ & $(4)$ & $(5)$ \\
Abell 1942 & 4961 & ACIS-I & 51.3 & 1.2 \\
Abell 2125 & 3290 & ACIS-I & 57.5 & 2.2 \\
MS 1455.0+2232 & 2207 & ACIS-I & 81.5 & 1.9 \\
ZwCl 1358.1+6245 & 4192 & ACIS-I & 91.9 & 1.8 \\
MS 1512.4+3647 & 516 & ACIS-S3 & 53.0 & 2.6 \\
\hline
\end{tabular}

Notes. Chandra Observation Log. Columns are: (1) cluster targeted; (2) Observation ID of Chandra data; (3) Detector used; (4) Usable exposure; (5) Estimate of the $2.0-8.0 \mathrm{keV}$ luminosity limit of the observation for a cluster galaxy.

Their data were telemetered and cleaned in Faint mode, and thus have a slightly higher background. As all observations were operated at $-120^{\circ} \mathrm{C}$ the X-ray data were corrected for the time dependence of the gain and the charge-transfer inefficiency with their photon energies determined using the gain file acisD200001-29gain_ctiN0006.fits. The archival data of all observations already had applied the newest tools to detect hot pixels and cosmic-ray afterglows. We only consider events with ASCA grades of $0,2,3,4$, and 6 . Known aspect offsets were applied for each observation. All observations were corrected for quantum efficiency degradation and had exposure maps determined at $1.5 \mathrm{keV}$. We excluded bad pixels, bad columns, and columns adjacent to bad columns or chip node boundaries. We also filtered out times when the blank-sky rate was more than three times the expected blank-sky rate derived from calibrated blanksky backgrounds to avoid the most extreme periods of high background ("background flares") that Chandra may encounter. MS 1512.4+3647 had two separate pointings and this introduced difficulties into our standard processing. We, therefore, excluded the shorter second pointing, which accounted for less than $25 \%$ of the total integration time.

To detect X-ray sources that are potential X-ray AGNs in these clusters, we applied the wavelet-detection algorithm (CIAO WAVDETECT) with scales ranging from 1 to 64 pixels in steps of $\sqrt{2}$ factors and required a source detection threshold of $10^{-6}$. Source detection was only performed in regions with an exposure of greater than $10 \%$ of the total for the observation. Our source detection threshold corresponds to $\lesssim 4$ falsely detected X- 
ray sources (due to a statistical fluctuation) for each observation. Using Kim et al. (2007), we have estimated the statistical X-ray positional uncertainty $(1 \sigma)$ due to WAVDETECT. In Table 4 , we list an estimated limiting X-ray luminosity for each observation that corresponds to five counts on axis (for consistency with Martini et al. 2006). For our analysis we concentrated on sources with at least 20 broad $(0.3-8.0 \mathrm{keV}) \mathrm{X}$-ray counts. These sources are unlikely to be due to statistical fluctuations except where they are coincident with ICM emission.

We used ACIS Extract $3.131^{8}$ to create source extraction regions enclosing $90 \%$ of the flux in the X-ray point-spread function (PSF) and to determine a masking radius that encircled $97 \%$ of the flux. For most of the sources, whose photons had median energies of $\sim 0.6-2.6 \mathrm{keV}$, we determined the regions assuming the PSF at $1.497 \mathrm{keV}$. A few sources had harder emission and their PSF was calculated assuming an energy of $4.51 \mathrm{keV}$. In a relatively small number of crowded regions, the PSF fraction was reduced to prevent overlapping source extraction regions. We also used ACIS Extract to correct the (CIAO WAVDETECT) position to the mean position of detected events for sources within $5^{\prime}$ of the observation aimpoint or to the position that best correlated with the PSF for sources beyond $5^{\prime}$ of the observation aimpoint. These new positions were registered with an optical catalog from $R$-band images (see below) to correct the absolute astrometry and determine the absolute astrometric precision of each Chandra observation $(0,3-0.5)$. The statistical significance of each detection was added in quadrature with the absolute astrometric precision to estimate the total X-ray positional precision. We measured the counts in three energy ranges: the broad $(0.3-8 \mathrm{keV})$, soft $(0.3-$ $2 \mathrm{keV})$, and hard $(2.0-8.0 \mathrm{keV})$ bands. The observed fluxes in these bands were derived assuming a $\Gamma=1.7$ power-law spectrum with Galactic absorption. We then calculated the rest-frame luminosity in the broadband $(0.3-8 \mathrm{keV})$ and the classic hardband (2-10 keV) for all sources with redshifts (see Section 3.3).

\subsection{MDM Photometry}

$R$-band images of these clusters were obtained at the MDM Observatory $2.4 \mathrm{~m}$ Hiltner telescope with the Echelle CCD camera during a run from the night of 2007 May 28 to 2007 June 3. Because the FOV of the CCD camera $(\sim 9.5 \times 9.5)$ is smaller than the ACIS-I FOV $\left(\sim 17^{\prime} \times 17^{\prime}\right)$, we imaged a $2 \times 2$ mosaic to cover the Chandra area, with each panel consisting of $3 \times 300$ s exposures. All images were trimmed, bias-subtracted and flat-fielded with the CCDPROC package within IRAF. ${ }^{9}$

Sources were cataloged with the SExtractor package (Bertin $\&$ Arnouts 1996). Aperture magnitudes from these catalogs were calibrated with multiple observations of standard star fields from the data compiled by P. B. Stetson ${ }^{10}$ onto the Vega magnitude system. Only data from the last night, which includes each quadrant of Abell 1240 and $\mathrm{ZwCl} 1358.1+6245$, the northeast quadrant of MS $1512.4+3647$, and $1 \times 300 \mathrm{~s}$ exposures of each quadrant of Abell 2125, were taken under photometric conditions. Our derived photometric solution for this night was precise to $0.03 \mathrm{mag}$. As all of these clusters

\footnotetext{
8 http://www.astro.psu.edu/xray/docs/TARA/ae_users_guide.html

9 IRAF is distributed by the National Optical Astronomy Observatories, which is operated by the Association of Universities for Research in Astronomy (AURA) under cooperative agreement with the National Science Foundation.

$10 \mathrm{http} / / /$ cadcwww.hia.nrc.ca/standards
}

except for Abell 2125 were imaged with SDSS, we crosscorrelated aperture magnitudes from all images on this run with stars in the SDSS DR5 catalog. After correcting to R (Vega), ${ }^{11}$ our derived photometric solution for June 3 , which includes a color-correction term, is accurate to $0.01 \mathrm{mag}$ and precise to $0.06 \mathrm{mag}$. The poorer precision compared to our photometric solution appears to be only partially due to the dispersion in the Vega correction (overlapping sources between quadrants of our own observations indicate typical photometric precisions of 0.05-0.08 mag). We, therefore, adopted the SDSS crosscalibration technique to photometrically correct all observations on non-photometric nights, except for observations of Abell 2125. For Abell 2125, nonphotometric observations were crosscalibrated with the single photometric exposures for Abell 2125. As we do not have complete multiband data, we report only the magnitudes assuming no color correction. The exclusion of the color-correction term does not significantly decrease the precision of our photometric solutions.

We calculated astrometric solutions for the images with the WCSTools package (Mink 2002), package and then produced the final, calibrated mosaics with the SWARP ${ }^{12}$ package. A final source catalog was extracted with SExtractor and used to register the astrometry of the X-ray observations. We consider only the SExtractor AUTO magnitudes, which is an automatic aperture magnitude designed to give precise estimates of total magnitudes for galaxies. As nearby, detected neighbors are removed and replaced by mirroring the opposite side of the aperture where available, these magnitudes are suitable for our relatively crowded fields. All X-ray sources that would be more luminous than $L_{X, H}=10^{43} \mathrm{erg} \mathrm{s}^{-1}$ at the cluster redshift that were also associated with galaxies and that would be more luminous than $M_{R}^{*}(z)+1$ at the cluster redshift were then targeted for the highest priority spectroscopic observations, with the exception of sources heavily contaminated by ICM emission. We also identified other candidate cluster X-ray AGNs, specifically those that would have $L_{X, H} \geqslant 10^{42} \mathrm{erg} \mathrm{s}^{-1}$, as lower-priority spectroscopic targets.

\subsection{MDM Spectroscopy}

We obtained low-resolution spectroscopy of these candidates with the $2.4 \mathrm{~m}$ Hiltner telescope with the CCDS, a Boller \& Chivens spectrograph, during a run from the night of 2008 April 28 to 2008 May 3. The slit widths were determined by the nightly seeing conditions and were either 1 1.'0 or 1".5. At least two exposures of every candidate were obtained and total exposure times varied from $120 \mathrm{~s}$ to $9000 \mathrm{~s}$. Five sets of internal and twilight flats were taken over the entire run, while comparison lamps were observed before and/or after every candidate.

The files were trimmed and bias-subtracted with the ccdproc package within IRAF and bad pixels were determined from a ratio of flat-field images and were fixed in every image. The individual flat-field images from internal lamps revealed a complex wavelength and slit-dependent flat field, most likely due to some reflection. To model this complex response, we first median-smoothed the internal flat fields (over $11 \times 11$ pixels) and then Gaussian-smoothed ( $\sigma=11$ pixels) over the dispersion axis. The ratio of the internal flat field to the modeled internal flat field was adopted as the true internal flat field. An illumination correction was then created from the twilight flat fields and

\footnotetext{
11 http://www.sdss.org/dr7/algorithms/sdssUBVRITransform.html\# Lupton2005

$12 \mathrm{http}: / /$ terapix.iap.fr/rubrique.php?id_rubrique $=49$
} 
Table 5

New, Lower-Luminosity Cluster X-ray AGNs

\begin{tabular}{|c|c|c|c|c|c|c|c|c|c|c|}
\hline $\begin{array}{c}\text { CXOU ID } \\
(1) \\
\end{array}$ & $\begin{array}{c}z \\
(2) \\
\end{array}$ & $\begin{array}{c}z \text { ref } \\
(3) \\
\end{array}$ & $\begin{array}{c}R \\
(4) \\
\end{array}$ & $\begin{array}{c}R \text { flag } \\
(5)\end{array}$ & $\begin{array}{c}f_{X, S} \\
(6) \\
\end{array}$ & $\begin{array}{c}f_{X, H} \\
(7)\end{array}$ & $\begin{array}{c}f_{X, B} \\
(8) \\
\end{array}$ & $\begin{array}{c}L_{X, B} \\
(9)\end{array}$ & $\begin{array}{c}L_{X, H} \\
(10) \\
\end{array}$ & $\begin{array}{c}\text { X flag } \\
(11) \\
\end{array}$ \\
\hline $\mathrm{J} 135950.5+623106.3$ & $0.32717 \pm 0.00038$ & 1 & $17.80 \pm 0.05$ & 3 & $9.5_{-1.0}^{+1.1}$ & $8.7_{-2.4}^{+2.8}$ & $20.6_{-2.1}^{+2.2}$ & $70.6_{-7.2}^{+7.6}$ & $46.2_{-4.7}^{+5.0}$ & 1 \\
\hline $\mathrm{J} 143821.8+034013.3$ & 0.22479 & 2 & $16.44 \pm 0.06$ & 3 & $2.20_{-0.76}^{+0.91}$ & $2.3_{-1.4}^{+1.9}$ & $4.8_{-1.5}^{+1.7}$ & $7.2_{-2.2}^{+2.5}$ & $4.7_{-1.4}^{+1.7}$ & 1 \\
\hline $\mathrm{J} 145714.7+221933.6$ & $0.24852 \pm 0.00025$ & 1 & $20.04 \pm 0.07$ & 0 & $2.40_{-0.53}^{+0.63}$ & $3.7_{-1.1}^{+1.3}$ & $5.9_{-1.0}^{+1.2}$ & $11.2_{-2.0}^{+2.2}$ & $7.3_{-1.3}^{+1.5}$ & 0 \\
\hline $\mathrm{J} 145715.0+222034.5$ & $0.25772 \pm 0.00015$ & 1 & $16.82 \pm 0.07$ & 0 & $20.2_{-4.8}^{+4.9}$ & $21.6_{-8.3}^{+8.5}$ & $44.4_{-9.0}^{+9.1}$ & $93_{-19}^{+19}$ & $61_{-12}^{+12}$ & 1 \\
\hline $\mathrm{J} 151422.5+363620.7$ & 0.3718 & 3 & $18.05 \pm 0.06$ & 2 & $3.98_{-0.89}^{+0.98}$ & $3.2_{-1.9}^{+2.5}$ & $8.4_{-1.8}^{+1.9}$ & $38.1_{-8.0}^{+8.8}$ & $24.9_{-5.2}^{+5.7}$ & 1 \\
\hline $\mathrm{J} 154101.9+661627.1$ & $0.24564 \pm 0.00045$ & 1 & $17.19 \pm 0.08$ & 2 & $2.78_{-0.52}^{+0.62}$ & $0.21_{-0.41}^{+0.76}$ & $4.63_{-0.87}^{+1.0}$ & $8.5_{-1.6}^{+1.9}$ & $5.5_{-1.0}^{+1.2}$ & 0 \\
\hline $\mathrm{J} 154101.9+661721.4$ & 0.2567 & 4 & $19.36 \pm 0.08$ & 0 & $8.11_{-0.88}^{+0.97}$ & $7.1_{-1.4}^{+1.7}$ & $17.0_{-1.6}^{+1.8}$ & $34.3_{-3.3}^{+3.6}$ & $22.4_{-2.1}^{+2.3}$ & 0 \\
\hline $\mathrm{J} 154117.3+661923.6$ & 0.2465 & 4 & $18.81 \pm 0.08$ & 0 & $2.08_{-0.47}^{+0.58}$ & $1.46_{-0.82}^{+1.2}$ & $4.15_{-0.88}^{+1.1^{0}}$ & $7.6_{-1.6}^{+1.9}$ & $5.0_{-1.1}^{+1.3}$ & 0 \\
\hline
\end{tabular}

Notes. Lower-luminosity AGN in the new, low-redshift cluster sample. Columns are: (1) name of X-ray source; (2) Redshift (3) References for redshift are: 1: this work; 2: SDSS (Adelman-McCarthy 2008); 3: Abraham et al. (1998); 4: Miller et al. (2004); (4) $R$-band magnitude; (5) Flags for photometry are: (0) no flag; (1) may be contaminated by nearby neighbors or bad pixels; (2) blended with nearby neighbors; (3) both; (6-8) Soft [0.3-2 keV], Hard [2-8 keV], and Broad [0.3-8 keV] band flux in the observed frame in units of $10^{-15} \mathrm{erg} \mathrm{s}^{-1} \mathrm{~cm}^{-2}$. (9-10) Broad $[0.3-8 \mathrm{keV}]$ and Hard [2-10 keV] band luminosity in the rest frame in units of $10^{41} \mathrm{erg} \mathrm{s}^{-1}$ corrected for Galactic absorption. (11) X-ray flags are: 0; no flag; 1; contaminated by ICM peak. Note that CXOU J145715.0+222034.5 is the BCG and we subtracted a multicomponent beta model for the ICM to compute the quoted fluxes and luminosities.

applied to make the final set of flat-field corrections to remove fringing in the spectra. After each spectrum was properly flat-fielded, we rejected cosmic rays using L.A. Cosmic ${ }^{13}$ (van Dokkum 2001). A fourth order wavelength solution was calculated for each set of $\mathrm{HgNe}$ comparison spectra, resulting in a typical rms of $\sim 0.1 \AA$ pixel $^{-1}$. Thereafter, standard aperture extraction of the spectra was used to remove the night sky emission and produce one-dimensional, logarithmically interpolated spectra with a dispersion of $\sim 3 \AA \mathrm{pixel}^{-1}$. The spectra extend from approximately $3650 \AA$ to $7250 \AA$. We extracted both the signal and noise for each final spectrum of a source.

We adapted the Princeton/MIT SDSS Spectroscopy routines ${ }^{14}$ to calculate redshifts. This technique cross-correlates the spectra in pixel space with template spectra, with each pixelweighted by the inverse of its variance, and is similar to the technique used in Martini et al. (2006). The template spectra include a set of four eigenspectra for galaxies, four eigenspectra for quasars, and 40 eigenspectra for stars. The five best galaxy redshifts for $-0.01<z<1.00$, five best quasar redshifts for $0.0033<z<7.00$, and 40 different stellar redshifts for $-0.004<z<0.004$ are found and ordered by the reduced $\chi^{2}$ of their fit. We adopted the best-fit redshift and classification for each source. To ascertain the quality of the fit and the errors to the redshift, we resampled each spectra 100 times randomly according to its noise characteristics and reran the cross-correlation routine. Both the dispersion in best-fit redshifts and the best-fit spectral type were used to qualify the spectral classification quality. If the dispersion in redshift was relatively low $\left(\sigma_{z} \lesssim 0.01\right),>68 \%$ of the best-fit redshifts were within $3 \sigma_{z}$ of our adopted redshift, and had the same spectral type (i.e., galaxy, quasar, or a similar stellar type) we consider this a secure redshift. Typically the maximum SNR of these spectra were $>5$ pixel $^{-1}$.

We did not identify any AGN in these clusters with $L_{X, H} \geqslant$ $10^{43} \mathrm{erg} \mathrm{s}^{-1}$, although we did identify several lower-luminosity AGNs in these clusters. Data for the lower-luminosity X-ray sources are provided in Table 5 and include several sources with spectroscopic measurements from the literature. The

\footnotetext{
$13 \mathrm{http} / / /$ www.astro.yale.edu/dokkum/lacosmic/

$14 \mathrm{http}: / /$ spectro.princeton.edu/idlspec2d_doc.html
}

spectroscopic observations of Abell 1240 and MS1512.4+3647 are complete for all candidates that would have $L_{X, H} \geqslant 10^{42}$ if at the cluster redshift, while the other four clusters are not complete to this luminosity limit. We have also measured redshifts, $R$-band magnitudes, and $\mathrm{X}$-ray fluxes and luminosities for numerous additional sources not associated with these clusters and their properties are listed in Table 6. As for the high-redshift clusters, several of the low-redshift clusters do not have direct velocity dispersion measurements. For Abell 1942 we estimated this quantity from the X-ray temperature. For Abell 1240 Xue $\& \mathrm{Wu}$ (2000) quote $k T=3.83 \mathrm{keV}$ from (Mushotzky \& Scharf 1997), but in fact the value in Mushotzky \& Scharf (1997) appears instead to be for Abell 1242. As we could not identify another $T_{X}$ value in the literature, we used the measurement of $L_{\text {bol }}=2.71 \times 10^{44} \mathrm{erg} \mathrm{s}^{-1}$ from David et al. (1999) and the relation $\sigma=10^{2.76} L_{X}^{0.19}$ derived by Xue \& Wu (2000) to estimate the velocity dispersion.

\section{CLUSTER X-RAY AGN FRACTION}

We require two quantities to estimate the AGN fraction in these clusters: the number of AGNs above our hard X-ray luminosity threshold hosted by galaxies with $M_{R}<M_{R}^{*}(z)+1$ and the total number of cluster galaxies above this magnitude threshold. For our low-redshift cluster sample, we have complete data to our X-ray threshold and reasonably complete data for the other cluster galaxies for about half of the clusters. For the high-redshift sample we have incomplete knowledge of both quantities. The AGN sample is likely incomplete because of spectroscopic incompleteness in the ChaMP and SEXSI surveys. The census of other cluster galaxies is very incomplete because few very high redshift clusters have the same quality membership data as our low-redshift sample. In the first three subsections below we describe the choice of the fiducial absolute magnitude threshold, our estimate of the completeness of the spectroscopic observations of X-ray sources, and the total number of cluster galaxies in the clusters with incomplete membership data. The fourth subsection describes our main result, the measurement of the AGN fraction and its evolution. The final two subsections describe potential contamination by AGNs associated with large-scale structure around these clusters and other sources of uncertainty, respectively. 
Table 6

Nonmember X-ray Sources

\begin{tabular}{|c|c|c|c|c|c|c|c|c|c|}
\hline $\begin{array}{c}\text { CXOU ID } \\
(1)\end{array}$ & $\begin{array}{c}z \\
(2) \\
\end{array}$ & $\begin{array}{c}z \text { ref } \\
(3)\end{array}$ & $\begin{array}{c}R \\
(4) \\
\end{array}$ & $\begin{array}{c}R \text { flag } \\
(5)\end{array}$ & $\begin{array}{c}f_{X, S} \\
(6) \\
\end{array}$ & $\begin{array}{c}f_{X, H 8} \\
(7) \\
\end{array}$ & $\begin{array}{c}f_{X, B} \\
(8) \\
\end{array}$ & $\begin{array}{c}\log L_{X, B} \\
(9)\end{array}$ & $\begin{array}{c}\log L_{X, H} \\
(10) \\
\end{array}$ \\
\hline $\mathrm{J} 112314.9+431208.3$ & $0.08017 \pm 0.00010$ & 1 & $17.66 \pm 0.08$ & 0 & $8.4_{-1.3}^{+1.5}$ & $29.9_{-4.1}^{+4.7}$ & $30.1_{-3.1}^{+3.4}$ & $41.69_{-0.04}^{+0.05}$ & $41.50_{-0.04}^{+0.05}$ \\
\hline $\mathrm{J} 112357.4+431314.1$ & 0.08007 & 2 & $19.46 \pm 0.08$ & 0 & $23.8_{-2.2}^{+2.3}$ & $32.8_{-4.3}^{+4.9}$ & $55.9_{-42}^{+4.5}$ & $44.51_{-0.03}^{+0.03}$ & $44.32_{-0.03}^{+0.03}$ \\
\hline $\mathrm{J} 112403.0+431330.6$ & 1.1049 & 2 & $18.39 \pm 0.08$ & 0 & $22.2_{-2.2}^{+2.2^{2}}$ & $17.3_{-3.6}^{+4.3}$ & $44.5_{-4.0}^{+4.4}$ & $43.16_{-0.04}^{+0.04}$ & $42.98_{-0.04}^{+0.04}$ \\
\hline $\mathrm{J} 112413.1+430639.3$ & $2.3666 \pm 0.0015$ & 1 & $19.80 \pm 0.08$ & 0 & $7.53_{-1.1}^{+1.2}$ & $7.56_{-2.0}^{+2.0}$ & $16.1_{-2.1}^{+2.4}$ & $44.73_{-0.06}^{+0.04}$ & $44.54_{-0.06}^{+0.04}$ \\
\hline $\mathrm{J} 143804.9+033752.6$ & $0.29192 \pm 0.00030$ & 1 & $18.50 \pm 0.06$ & 0 & $5.22_{-0.95}^{+1.1}$ & $<4.0$ & $7.8_{-1.5}^{+1.8}$ & $42.32_{-0.09}^{+0.106}$ & $42.13_{-0.09}^{+0.10}$ \\
\hline $\mathrm{J} 143832.2+033506.0$ & $1.0083 \pm 0.0051$ & 1 & $19.98 \pm 0.06$ & 0 & $65.7_{-3.0}^{+3.2}$ & $79.8_{-5.8}^{+6.2}$ & $149.3_{-5.8}^{+6.0}$ & $44.85_{-0.02}^{+0.02}$ & $44.66_{-0.02}^{+0.02}$ \\
\hline $\mathrm{J} 143833.0+033606.8$ & $0.38252 \pm 0.00017$ & 1 & $19.40 \pm 0.06$ & 0 & $11.5_{-1.2}^{+1.3}$ & $18.3_{-2.7}^{+3.1}$ & $28.5_{-2.4}^{+2.6}$ & $43.15_{-0.04}^{+0.04}$ & $42.96_{-0.04}^{+0.04}$ \\
\hline $\mathrm{J} 143839.7+033631.3$ & $2.1493 \pm 0.0019$ & 1 & $19.00 \pm 0.06$ & 0 & $16.1_{-1.5}^{+1.7^{2}}$ & $16.1_{-2.6}^{+3.1}$ & $34.8_{-2.8}^{+3.4}$ & $44.97_{-0.04}^{+0.04}$ & $44.79_{-0.04}^{+0.04}$ \\
\hline $\mathrm{J} 143841.9+034110.2$ & 1.7372 & 2 & $17.82 \pm 0.06$ & 0 & $28.2_{-2.2}^{+2.5}$ & $29.5_{-3.8}^{+4.6}$ & $61.6_{-4.1}^{+4.8}$ & $45.01_{-0.03}^{-0.03}$ & $44.83_{-0.03}^{+0.03}$ \\
\hline $\mathrm{J} 143847.3+032950.8$ & $0.00034 \pm 0.00012$ & 1 & $16.89 \pm 0.06$ & 0 & $16.3_{-1.8}^{+2.2}$ & $2.0_{-1.6}^{+2.2^{\circ}}$ & $26.9_{-3.0}^{+3.1}$ & & \\
\hline $\mathrm{J} 143859.0+033547.8$ & 0.7339 & 2 & $18.51 \pm 0.06$ & 0 & $46.5_{-2.7}^{+2.8}$ & $7.1_{-5.7}^{+6.0}$ & $113.8_{-5.3}^{+5.6}$ & $44.41_{-0.02}^{+0.02}$ & $44.22_{-0.02}^{+0.02}$ \\
\hline $\mathrm{J} 145623.0+221833.5$ & $0.00027 \pm 0.00010$ & 1 & $15.51 \pm 0.07$ & 0 & $9.0_{-1.3}^{+1.5}$ & $5.1_{-2.0}^{+2.5}$ & $17.1_{-2.3}^{+2.63}$ & & \\
\hline $\mathrm{J} 145624.5+222057.1$ & $0.00019 \pm 0.00010$ & 1 & $15.45 \pm 0.07$ & 0 & $14.8_{-1.3}^{+1.4}$ & $11.3_{-2.2}^{+2.5}$ & $29.8_{-2.4}^{+2.5}$ & & \\
\hline $\mathrm{J} 145634.6+221514.2$ & $0.40918 \pm 0.00010$ & 1 & $20.16 \pm 0.07$ & 0 & $25.8_{-1.6}^{+1.7^{3}}$ & $56.8_{-4.1}^{+4.2}$ & $73.3_{-3.5}^{+3.4}$ & $43.63_{-0.02}^{+0.02}$ & $43.45_{-0.02}^{+0.02}$ \\
\hline $\mathrm{J} 145657.7+221315.6$ & $0.00016 \pm 0.00010$ & 1 & $14.87 \pm 0.07$ & 0 & $8.63_{-0.93}^{+1.0^{\circ}}$ & $3.6_{-1.1}^{+1.4}$ & $16.0_{-16}^{+1.3}$ & & \\
\hline $\mathrm{J} 145708.7+222352.4$ & 0.1238 & 2 & $17.44 \pm 0.07$ & 0 & $2.27_{-0.50}^{+0.60}$ & $<3.4$ & $3.32_{-0.86}^{+1.0}$ & $41.14_{-0.11}^{+0.13}$ & $40.95_{-0.11}^{+0.13}$ \\
\hline $\mathrm{J} 145710.7+221844.9$ & $1.885 \pm 0.0014$ & 1 & $18.73 \pm 0.07$ & 0 & $3.99_{-0.57}^{+0.66}$ & $5.3_{-1.1}^{+1.4}$ & $9.4_{-1.1}^{+1.20}$ & $44.28_{-0.05}^{+0.06}$ & $44.09_{-0.05}^{+0.06}$ \\
\hline $\mathrm{J} 145712.3+221446.7$ & $-0.00069 \pm 0.00010$ & 1 & $15.15 \pm 0.07$ & 1 & $50.4_{-2.1}^{+2.1}$ & $15.3_{-2.0}^{+2.1}$ & $90.2_{-3.5}^{+3.16}$ & & \\
\hline $\mathrm{J} 145721.0+222334.5$ & $1.7362 \pm 0.0010$ & 1 & $19.33 \pm 0.07$ & 1 & $9.4_{-10}^{+1.1}$ & $7.0_{-16}^{+1.9}$ & $18.9_{-1.9}^{+2.0}$ & $44.50_{-0.04}^{+0.05}$ & $44.32_{-0.04}^{+0.05}$ \\
\hline $\mathrm{J} 145726.9+221755.1$ & $1.4664 \pm 0.0011$ & 1 & $19.55 \pm 0.07$ & 0 & $23.6_{-1.6}^{+1.9}$ & $33.0_{-3.1}^{+3.0}$ & $56.3_{-3.1}^{+3.2}$ & $44.81_{-0.02}^{+0.03}$ & $44.62_{-0.02}^{+0.03}$ \\
\hline $\mathrm{J} 151427.0+363803.1$ & 0.1616 & 2 & $16.90 \pm 0.06$ & 0 & $2.28_{-0.49}^{+0.61}$ & $1.8_{-1.1}^{+1.9}$ & $4.82_{-0.99}^{+3.1}$ & $41.53_{-0.09}^{+0.02}$ & $41.35_{-0.09}^{+0.11}$ \\
\hline $\mathrm{J} 151428.4+363743.5$ & 0.4026 & 3 & $20.13 \pm 0.06$ & 0 & $7.70_{-0.92}^{+1.0}$ & $14.1_{-3.0}^{+3.1}$ & $18.9_{-2.0}^{+2.29}$ & $43.01_{-0.05}^{+0.05}$ & $42.83_{-0.05}^{+0.05}$ \\
\hline $\mathrm{J} 151437.5+364041.3$ & 0.1468 & 3 & $19.86 \pm 0.06$ & 0 & $12.1_{-1.1}^{+1.22}$ & $13.5_{-2.9}^{+3.5}$ & $26.9_{-2.3}^{+2.4}$ & $42.19_{-0.04}^{+0.04}$ & $42.01_{-0.04}^{+0.04}$ \\
\hline $\mathrm{J} 153938.1+662102.4$ & 0.4375 & 4 & $19.71 \pm 0.08$ & 0 & $5.02_{-0.98}^{+1.1}$ & $6.5_{-2.4}^{+2.9}$ & $11.7_{-2.1}^{+2.3}$ & $42.90_{-0.08}^{+0.09}$ & $42.71_{-0.08}^{+0.09}$ \\
\hline $\mathrm{J} 154012.3+661439.2$ & $1.0577 \pm 0.0029$ & 1 & $19.75 \pm 0.08$ & 0 & $37.2_{-1.8}^{+1.9}$ & $41.9_{-3.4}^{+3.4}$ & $83.1_{-3.5}^{+3.1}$ & $44.64_{-0.02}^{+0.02}$ & $44.46_{-0.02}^{+0.02}$ \\
\hline
\end{tabular}

Notes. Nonmembers from the new, low-redshift cluster sample. Columns are: Col (1) Name of X-ray source; Col (2) Redshift; Col (3) References for redshift are: 1: this work; 2: SDSS (Adelman-McCarthy 2008); 3: Abraham et al. (1998); 4: Miller et al. (2004); Col (4) $R$-band magnitude; Col (5) Flags for photometry are: 0; no flag; 1; may be contaminated by nearby neighbors or bad pixels; Cols (6-8) Soft [0.5-2 keV], Hard [2-8 keV], and Broad [0.5-8 keV] band flux in the observed frame in units of $10^{-15} \mathrm{erg} \mathrm{s}^{-1} \mathrm{~cm}^{-2}$. Upper limits are $3 \sigma$ limits. Cols (9-10) Log of the Broad [0.5-8 $\mathrm{keV}]$ and Hard [2-10 keV] band luminosity in the rest-frame in units of $\mathrm{erg} \mathrm{s}^{-1}$ corrected for Galactic absorption. We do not quote luminosities for $\mathrm{X}$-ray sources identified with Galactic stars $(z \sim 0)$.

\subsection{Host-Galaxy Magnitude Threshold}

In previous work we defined the AGN fraction in clusters relative to galaxies more luminous than an $R$-band absolute magnitude of $M_{R}=-20$ mag (e.g., Martini et al. 2006). This choice of magnitude threshold was largely driven by expedience, namely it corresponded to the completeness limit for the most distant clusters in that sample. To properly extend this work to high redshift it is important to account for the evolution of the galaxy population in clusters, both in luminosity and number. These were not significant effects in our low-redshift study as the highest-redshift cluster was at only $z=0.31$, but in our previous work at $z \sim 0.6$ by Eastman et al. (2007) the $M_{R}=-20$ mag cutoff corresponded to a fainter absolute magnitude relative to $M_{R}^{*}$. Because the cluster galaxy population is larger, this would have led to a lower estimate of the AGN fraction if the cluster AGNs are predominantly associated with the most luminous galaxies, as is the case at low redshifts (Sivakoff et al. 2008).

Here we adopt an absolute magnitude threshold of $M_{R}^{*}(z)+1$, and thus allow for evolution of $M_{R}^{*}$. At low-redshifts $(0.01<$ $z<0.07$ ) Christlein \& Zabludoff (2003) measured the $R$-band luminosity function (LF) for six nearby clusters ${ }^{15}$ and found that the composite cluster LF is consistent with a Schechter function with $M_{R}^{*}=-21.92 \pm 0.17 \mathrm{mag}(h=0.7, \alpha=-1.21)$. They also found an essentially identical value of $M_{R}^{*}=-21.93 \mathrm{mag}$ for the field. The low-redshift value of $M_{R}^{*}+1$ is therefore about

\footnotetext{
15 Two of these clusters (Abell 85 and Abell 754) are in our low-redshift sample (Sivakoff et al. 2008).
}

one magnitude brighter than the value of $M_{R}=-20$ mag we adopted in our previous, low-redshift studies (Martini et al. 2006; Sivakoff et al. 2008). For comparison, Blanton et al. (2003) measured $M^{*}=-21.22(\alpha=-1.05)$ at $z=0.1$ for the $r^{0.1}$ band on the $\mathrm{AB}$ system. This corresponds to $M_{R}^{*}=-21.72 \mathrm{mag}$ on the Vega system for the $R$ band at $z=0$ based on the conversions presented in Blanton \& Roweis (2007) and is therefore consistent with Christlein \& Zabludoff (2003).

Many recent studies have measured the evolution of $M_{R}^{*}$ and generally these measurements include both a value for all galaxies and separate measurements for particular spectroscopic types. This has relevance for our study as the cluster galaxy population is on average more quiescent than field galaxies and consequently their evolutionary history is different. We are most interested in measurements of the evolution of $M_{R}^{*}$ as a function of spectral type to isolate the evolution of galaxies dominated by older stellar populations that are most likely representative of the evolution of cluster galaxies. A useful, low-redshift benchmark for a type-dependent LF for clusters comes again from Christlein \& Zabludoff (2003). They found $M_{R}^{*}=-21.78 \mathrm{mag}$ for quiescent galaxies in clusters, which is nearly identical to the value for all cluster members. For field galaxies Chen et al. (2003) use photometric redshifts in the Las Campanas Infrared Survey and measure values of -21.70 to $-22.22 \mathrm{mag}(\alpha=-1)$ for all galaxies over the range $0.5<z<1.5$ and values of -21.21 to $-21.82 \mathrm{mag}(\alpha=-0.2)$ for galaxies consistent with an $\mathrm{E} / \mathrm{S} 0+\mathrm{Sab}$ spectral template. Wolf et al. (2003) used photometric redshifts from COMBO-17 
and measured more pronounced evolution for their early-type spectral template with $M_{R}^{*}$ fading by $\sim 1$ mag from $z \sim 1.1$ to $z \sim 0.3$. More recently, Ilbert et al. (2005) measured a fading of 1.1-1.8 mag between $z \sim 2$ and $z \sim 0.1$ in the $R$ band based on spectroscopic redshifts, although they do not present the evolution as a function of spectral type. These measurements of evolution in $M_{R}^{*}$ are broadly comparable to the 1.2 mag of fading from $z=1$ to the present expected from pure luminosity evolution of a single stellar population with $z_{f}=2$ and solar metallicity (Bruzual \& Charlot 2003).

Direct measurements of evolution of the cluster LF have mostly been conducted in the rest-frame $B$ band. Goto et al. (2005) found $M_{B}^{*}=-21.13 \mathrm{mag}$ for MS1054-03 $(z=0.83)$, which is in our sample, and similar to the $M_{B}^{*}=-21.15 \mathrm{mag}$ measured for three clusters at an average $z=0.859$ by Postman et al. (2001). In comparison to local $B$-band measurements of the cluster LF (e.g., Colless 1989; Rauzy et al. 1998), Goto et al. (2005) concluded that $M_{B}^{*}$ fades by 0.46 to 0.71 mag between $z=0.83$ and $z=0$. For the same simple stellar population model considered above (Bruzual \& Charlot 2003), 1.2 mag of fading in $B$ band is expected from $z=0.83$ to the present. While there is not a direct measurement in the rest-frame $R$ band for the cluster LF, at yet longer wavelengths Ellis \& Jones (2004) found that the fading in the $K$ band is 1.2 mag from $z=0.9$ to the present and consistent with passive evolution and a formation epoch at $z_{f}=2$. From these investigations of the LF evolution in the field and clusters, we adopt the assumption that $M_{R}^{*}(z)=M_{R}^{*}(0)-z$ and the normalization for $M_{R}^{*}$ from (Christlein \& Zabludoff 2003) for all cluster galaxies to estimate the completeness of the spectroscopy of X-ray counterparts and the size of the galaxy population in low-redshift clusters. This result is broadly consistent with all of the results described here, although is most consistent with the studies that predict more fading. If there is less fading of galaxies at the bright end of the LF, such as may be due to some low-level star formation in these galaxies, then the completeness limits we describe next are too bright and we will have systematically underestimated the population of luminous AGNs in the higher-redshift clusters.

\subsection{Completeness}

We calculate a completeness limit in the observed $R$ band for each cluster based on the value of $M_{R}^{*}(z)+1$ and a $K$-correction derived from the elliptical template of the four-component spectral template presented by Assef et al. (2008). These templates are derived from 16,033 galaxies with spectroscopic redshifts and multiband photometry from the AGNs and Galaxy Evolution Survey. Most of the galaxies are in the range $0<$ $z<1$ and the median redshift is 0.31 . The parent sample is therefore broadly representative of our redshift range. For the higher-redshift clusters, the $K$-correction requires a substantial extrapolation from the observed $R$ band, which, for example, samples rest-frame $B$ band at $z=0.5$. Our assumption that the typical cluster galaxies are best approximated by an elliptical template is certainly reasonable for the low-redshift clusters. This may not be as good an approximation at higher redshifts, although in a study of the color-magnitude relation in our two highest redshift clusters (Lynx E and W) Mei et al. (2009) found there is no evidence for significant evolution. If a later-type template were a better choice for the $K$-correction at higher redshift, the $K$-correction would be smaller and the necessary $R$-band spectroscopic limit would be brighter. The net effect would be a smaller completeness correction.

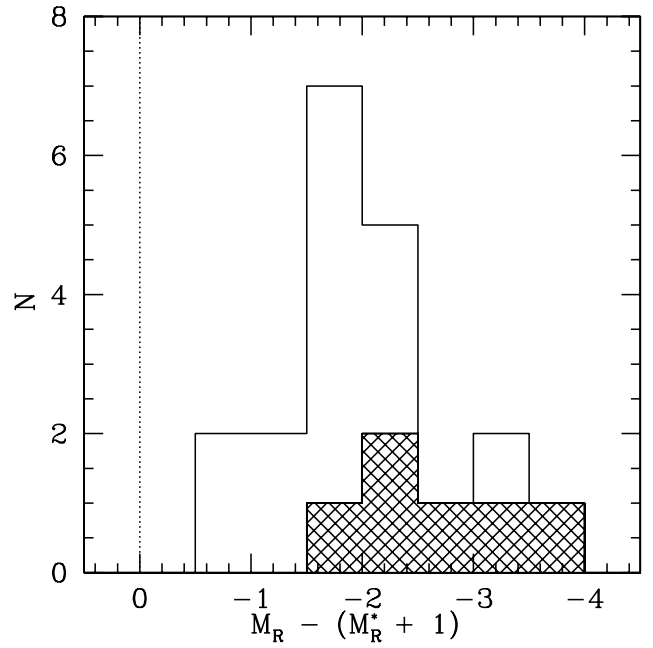

Figure 1. Distribution in absolute magnitude $M_{R}$ of the cluster AGNs relative to $M_{R}^{*}(z)+1$ at their redshift. All of the cluster AGNs are substantially brighter than $M_{R}^{*}(z)+1$, although in most cases the spectroscopy is complete to this limit. The subsets that are classified as BLAGN are represented by the hatched histogram. The dotted line corresponds to our galaxy luminosity threshold at $M_{R}^{*}(z)+1$.

The spectroscopic completeness of the high- $z$ AGN sample largely depends on the completeness of the ChaMP and SEXSI surveys, although we also use additional spectra for MS 2053.70449, MS 1054-03, and RDCS J0910+5422. The ChaMP survey quotes a spectroscopic completeness of $77 \%$ for $R<$ 22.37 mag (Silverman et al. 2005) and the SEXSI survey quotes a spectroscopic completeness of $61 \%$ for sources with $22 \mathrm{mag}<R<23 \mathrm{mag}, 67 \%$ for sources with $23 \mathrm{mag}<R<$ $24 \mathrm{mag}$, and $74 \%$ for sources with $R>24 \mathrm{mag}$ (typically to $24.4 \mathrm{mag}$; Eckart et al. 2006). For the ChaMP data we adopt $77 \%$ as the completeness correction for $R<22.37 \mathrm{mag}$, while for the SEXSI survey we adopt an average completeness correction of $67 \%$ for $R<24.4 \mathrm{mag}$. For nearly all of the clusters above $z>0.6$ the spectroscopic data do not extend to the equivalent of $M_{R}^{*}(z)+1$ and the size of the magnitude range without spectra ranges from a few tenths to over a magnitude. To estimate the number that may have been missed we inspected the host-galaxy absolute magnitude distribution of the $L_{X, H} \geqslant 10^{43}$ erg s ${ }^{-1}$ AGN in the clusters with complete data and find only one AGN fainter than $M_{R}^{*}$. The distribution in $M_{R}$ of the X-ray AGNs is shown in Figure 1. We, therefore, assume that we have not missed any AGN because the spectroscopic observations of $\mathrm{X}$-ray sources did not have the requisite depth, although this assumption may have led us to underestimate the AGN fraction at high redshift. In contrast, if our assumption of an early-type template for the $K$-correction was too red, then the spectroscopic data do achieve the requisite depth and this remains a nonissue. At brighter apparent magnitudes we do apply a completeness correction to account for the quoted $77 \%$ and $67 \%$ completeness of the surveys. We discuss this further in Section 4.4 below.

The X-ray AGN populations of several of these clusters have been studied in previous work. The first substantial study of spectroscopically confirmed X-ray AGN in highredshift clusters was by Johnson et al. (2003) in MS1054-03. They identified 2 AGNs associated with this cluster: CXOU J105702.7-033943 and CXOU J105710.6-033500; however, neither of these are included in the present sample. The first was not included because the X-ray luminosity is below our threshold of $10^{43} \mathrm{erg} \mathrm{s}^{-1}$ and the second because it falls slightly 
Table 7

AGN Fraction Estimates and Cluster Membership

\begin{tabular}{|c|c|c|c|c|c|c|c|}
\hline $\begin{array}{c}\text { Cluster } \\
(1) \\
\end{array}$ & $\begin{array}{c}z \\
(2) \\
\end{array}$ & $\begin{array}{c}\sigma \\
(3)\end{array}$ & $\begin{array}{c}N_{A G N} \\
(4)\end{array}$ & $\begin{array}{c}N_{\text {gal }} \\
(5)\end{array}$ & $\begin{array}{c}\text { Flag } \\
(6)\end{array}$ & $\begin{array}{c}f_{A, \text { raw }}(\%) \\
(7)\end{array}$ & $\begin{array}{c}f_{\text {spec }} \\
(8) \\
\end{array}$ \\
\hline Abell754 & 0.0546 & 953 & 1 & 82 & 1 & $1.2_{-1.0}^{+2.8}$ & $\overline{1.00}$ \\
\hline Abell85 & 0.0554 & 993 & 0 & 53 & 1 & $<2.2$ & 1.00 \\
\hline Abell3128 & 0.0595 & 906 & 0 & 28 & 1 & $<4.1$ & 1.00 \\
\hline Abell3125 & 0.0616 & 475 & 0 & 15 & 1 & $<7.7$ & 1.00 \\
\hline Abell644 & 0.0701 & 952 & 0 & 40 & 1 & $<2.9$ & 1.00 \\
\hline Abell89B & 0.0770 & 474 & 0 & 12 & 1 & $<9.6$ & 1.00 \\
\hline Abell2104 & 0.1544 & 1242 & 1 & 54 & 1 & $1.9_{-1.5}^{+4.3}$ & 1.00 \\
\hline Abell1240 & 0.1590 & 698 & 0 & 28 & 2 & $<4.1$ & 1.00 \\
\hline Abell1689 & 0.1867 & 1400 & 0 & 184 & 1 & $<0.62$ & 1.00 \\
\hline Abell2163 & 0.2007 & 1381 & 0 & 262 & 1 & $<0.44$ & 1.00 \\
\hline Abell1942 & 0.2240 & 905 & 0 & 65 & 2 & $<1.8$ & 1.00 \\
\hline Abell2125 & 0.2465 & 1113 & 0 & 127 & 2 & $<0.90$ & 1.00 \\
\hline MS1455.0+2232 & 0.2578 & 1032 & 0 & 99 & 2 & $<1.2$ & 1.00 \\
\hline MS1008.1-1224 & 0.3068 & 1127 & 0 & 216 & 1 & $<0.53$ & 1.00 \\
\hline AC114 & 0.3148 & 1388 & 0 & 121 & 1 & $<0.95$ & 1.00 \\
\hline ZwCl1358.1+6245 & 0.328 & 1003 & 0 & 91 & 2 & $<1.3$ & 1.00 \\
\hline MS1512.4+3647 & 0.372 & 575 & 0 & 15 & 2 & $<7.7$ & 1.00 \\
\hline MS1621.5+2640 & 0.430 & 735 & 0 & 65 & 2 & $<1.8$ & 0.67 \\
\hline $3 C 295$ & 0.460 & 1642 & 2 & 412 & 2 & $0.49_{-0.31}^{+0.64}$ & 0.67 \\
\hline MS0451.6-0305 & 0.538 & 1371 & 0 & 273 & 2 & $<0.42$ & 0.77 \\
\hline MS0015.9+1609 & 0.541 & 1234 & 1 & 214 & 2 & $0.47_{-0.39}^{+1.1}$ & 0.77 \\
\hline RXJ0848.7+4456 & 0.574 & 895 & 1 & 102 & 2 & $0.98_{-0.81}^{+2.3}$ & 0.67 \\
\hline MS2053.7-0449 & 0.583 & 865 & 0 & 95 & 2 & $<1.2$ & 1.00 \\
\hline RXJ0542.8-4100 & 0.634 & 1269 & 5 & 229 & 2 & $2.18_{-0.94}^{+1.5}$ & 0.77 \\
\hline RXJ2302.8+0844 & 0.722 & 658 & 0 & 50 & 2 & $<2.3$ & 0.77 \\
\hline MS1137.5+6625 & 0.782 & 885 & 1 & 100 & 2 & $1.00_{-0.83}^{+2.3}$ & 0.77 \\
\hline RX J1317.4+2911 & 0.805 & 1142 & 1 & 179 & 2 & $0.56_{-0.46}^{+1.3}$ & 0.67 \\
\hline RXJ1716.4+6708 & 0.813 & 1445 & 3 & 308 & 2 & $0.97_{-0.53}^{+0.95}$ & 0.92 \\
\hline MS 1054-03 & 0.823 & 1156 & 1 & 184 & 2 & $0.54_{-0.45}^{+1.2}$ & 0.77 \\
\hline RDCS J0910+5422 & 1.110 & 675 & 1 & 53 & 2 & $1.9_{-1.6}^{+4.3}$ & 0.67 \\
\hline Lynx E & 1.261 & 740 & 1 & 66 & 2 & $1.5_{-1.3}^{+3.5}$ & 0.67 \\
\hline Lynx W & 1.270 & 650 & 1 & 49 & 2 & $2.0_{-1.7}^{+4.7}$ & 0.67 \\
\hline
\end{tabular}

Notes. AGN fraction estimates for individual clusters. Columns are: Col. (1): Cluster name; Col. (2): Redshift; Col. (3): Velocity dispersion (references for these values are in Table 1, Table 3, Sivakoff et al. (2008) for Abell 754, Abell 85, Abell 89B, Martini et al. (2006) for Abell 3128, Abell 3125, Abell 644, Abell 2104, Abell 2163, and MS1008.1-1224, or adopted from Czoske (2004) for Abell 1689 and Girardi \& Mezzetti (2001) for AC 114); Col. (4): Number of AGN with $L_{X, H} \geqslant 10^{43} \mathrm{erg} \mathrm{s}^{-1}$ in galaxies more luminous than $M_{R}^{*}(z)+1$; Col. (5): Estimate of the number of cluster galaxies more luminous than $M_{R}^{*}(z)+1$ within either the Chandra FOV or $R_{200}$, whichever is smaller; Col. (6): Flag for the origin of the estimate where 1: from our spectroscopy and completeness correction; 2: from the MaxBCG as described in Section 4.3; Col. (7): Estimate of the cluster AGN fraction in percent; Col. (8): Estimate of the spectroscopic completeness for X-ray sources.

outside the projected virial radius $\left(R / R_{200}=1.2\right)$. Martel et al. (2007) have also studied X-ray sources in clusters, including three clusters that overlap this sample. They are discussed further in Section 5.3 below.

\subsection{Inactive Cluster Galaxy Population}

To estimate the AGN fraction in these clusters we need to know the number of cluster galaxies more luminous than $M_{R}^{*}(z)+1$. We estimate this quantity in two ways, depending on the available data for the clusters. For the low-redshift clusters in our previous studies (Martini et al. 2006, 2007; Sivakoff et al. 2008) we have a large number of spectroscopically confirmed cluster members and can estimate the number of cluster galaxies either directly or with a completeness correction. We have calculated new estimates for these clusters for the present paper because we no longer use the $M_{R}=-20$ mag threshold of the previous work. These values are listed in Table 7.

For essentially all of the new clusters in the present study we employ the same technique as Eastman et al. (2007) to estimate the number of cluster members above $M_{R}^{*}(z)+1$ from the cluster velocity dispersion. This employs the richnessvelocity dispersion relationship defined by Koester et al. (2007) for the MaxBCG cluster sample. The cluster richness $N_{\mathrm{gal}}^{R 200}$ is the number of red (E/S0) cluster members more luminous than $0.4 L^{*}$ within the projected $R_{200}$ radius. This relationship was originally derived from a sample of 13,823 clusters with $0.1<z<0.3$ in the SDSS with velocity dispersions greater than $\sim 400 \mathrm{~km} \mathrm{~s}^{-1}$. Becker et al. (2007) provided the most recent estimate of this relation based on a larger sample that extends over both a broader redshift range and to lower velocity dispersion groups. They found $\ln \sigma=(6.17 \pm 0.04)+(0.436 \pm$ $0.015) \ln N_{\text {gal }}^{R 200} / 25$. For reference, a $520 \mathrm{~km} \mathrm{~s}^{-1}$ cluster has $N_{\mathrm{gal}}^{R 200}=30$.

There are several caveats that need to be considered with the use of this estimator. First, the richness-velocity dispersion relationship is based on photometric and not spectroscopic redshifts. This is not a significant concern because for red cluster galaxies the photometric redshift estimates are robust within the quoted uncertainties. The second concern is that this relationship is based on the red cluster galaxies alone. At low redshifts this estimate is a reasonable approximation as the vast majority of cluster galaxies more luminous than $M_{R}^{*}+1$ fall in this category. For example, the fraction of quiescent galaxies above this luminosity in the composite LF of Christlein \& Zabludoff (2003) is $\sim 85 \%$. While their definition of quiescence is based on spectral lines rather than color, these two definitions of quiescence typically agree when averaged over a cluster. At higher redshifts, a larger fraction of the cluster galaxies may be blue due to ongoing star formation, but this cannot be a substantial contribution because the luminosity-weighted mean star formation epoch is $z=2$ for early-type cluster galaxies up to $z=0.5$ (van Dokkum \& van der Marel 2007). Becker et al. (2007) did find evidence of evolution in this relationship in the sense of lower richness at fixed velocity dispersion in higherredshift clusters, but they note that this may be due to their strict color selection. In addition, for our accounting of the inactive galaxy population the color of the galaxies does not matter so long as they are in the cluster and above the luminosity threshold. Observations of individual clusters with extensive spectroscopic data support the assumption that there is no substantial evolution in the relation between halo occupation number and cluster mass (Muzzin et al. 2007). This is also supported by several theoretical studies that find minimal evolution in the number of bright galaxies in massive halos (Kravtsov et al. 2004; Zentner et al. 2005).

We performed an independent validation of the MaxBCG relation with an analysis of the individual clusters in our sample with substantial membership data. While most of the lowredshift clusters have substantial membership data, these data generally do not extend to our estimate of $R_{200}$ (Martini et al. 2007), nor is the X-ray coverage complete to this radius. Our spectroscopic coverage was often limited to the size of the Chandra field of view. However, two useful exceptions are Abell 89B and MS1008.1-1224 and in both cases estimates agree to within a factor of 2. Our wide-field X-ray coverage of Abell 85 and Abell 754 (Sivakoff et al. 2008) was designed to sample 
Table 8

AGN Fraction for Subsamples of the Clusters

\begin{tabular}{|c|c|c|c|c|c|c|c|c|c|}
\hline $\begin{array}{c}\text { Sample } \\
(1)\end{array}$ & $\begin{array}{l}z \text { range } \\
(2) \\
\end{array}$ & $\begin{array}{c}N_{C L} \\
\text { (3) } \\
\end{array}$ & $\begin{array}{c}\text { median } z \\
(4)\end{array}$ & $\begin{array}{c}\text { median } \sigma \\
(5)\end{array}$ & $\begin{array}{c}N_{A, \text { raw }} \\
(6)\end{array}$ & $\begin{array}{c}N_{\text {gal }} \\
(7) \\
\end{array}$ & $\begin{array}{c}f_{A, \text { raw }}(\%) \\
(8)\end{array}$ & $\begin{array}{c}f_{\text {spec }} \\
(9) \\
\end{array}$ & $f_{A, \text { corr }}(\%)$ \\
\hline \multicolumn{10}{|l|}{ Two Bins } \\
\hline & $z<0.4$ & 17 & 0.19 & 993 & 2 & 1492 & $0.134_{-0.087}^{+0.18}$ & 1.00 & $0.134_{-0.087}^{+0.18}$ \\
\hline & $z>0.4$ & 15 & 0.72 & 895 & 18 & 2379 & $0.76_{-0.18}^{+0.22}$ & 0.76 & $1.00_{-0.23}^{+0.29}$ \\
\hline \multicolumn{10}{|l|}{ Three Bins } \\
\hline & $z<0.3$ & 13 & 0.15 & 953 & 2 & 1049 & $0.19_{-0.12}^{+0.25}$ & 1.00 & $0.19_{-0.12}^{+0.25}$ \\
\hline & $0.3<z<0.6$ & 10 & 0.45 & 1065 & 4 & 1604 & $0.25_{-0.12}^{+0.20}$ & 0.81 & $0.31_{-0.15}^{+0.24}$ \\
\hline & $z>0.6$ & 9 & 0.81 & 885 & 14 & 1218 & $1.15_{-0.30}^{+0.39}$ & 0.78 & $1.47_{-0.39}^{+0.50}$ \\
\hline
\end{tabular}

Notes. Cluster AGN fractions with the data split into two bins and three bins. The two bins are split at $z=0.4$, while the three bins split the data at $z=0.3$ and $z=0.6$. For each bin we list: Col. (2): redshift range; Col. (3): number of clusters; Col. (4): median redshift; Col. (5): median velocity dispersion of clusters; Col. (6): sum of the luminous AGN in the bin; Col. (7): raw AGN fraction with double-sided, $1 \sigma$ confidence limits; Col. (8): estimate of the mean spectroscopic completeness weighted by the number of galaxies per cluster; Col. (9): AGN fraction corrected for spectroscopic completeness.

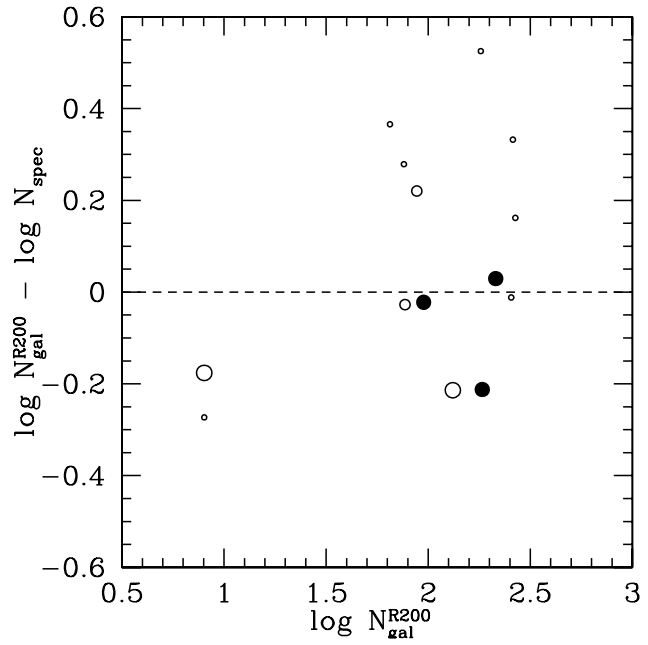

Figure 2. Difference between predicted and measured cluster richness compared to the cluster richness predicted by the MaxBCG sample. The quantity $N_{\mathrm{gal}}^{R 200}$ is the number of red cluster galaxies more luminous than $0.4 L^{*}$ and estimated from the cluster velocity dispersion (Becker et al. 2007), while $N_{\text {spec }}$ is a spectroscopic estimate of this quantity (see Section 4.3). Symbols are coded according to the spectroscopic completeness relative to $R_{200}$. The large circles have complete coverage to $R_{200}$, medium circles have more than $50 \%$ coverage, and the small circles have less than $50 \%$ coverage. Most clusters are at $z<0.5$ (open symbols), although substantial data exist for three at $z>0.5$ (filled symbols). See Section 4.3 for further details.

a substantial fraction of the projected $R_{200}$ and these values also agree well. Figure 2 illustrates the difference between the MaxBCG membership estimates and our spectroscopic estimates. The larger points have nearly complete spectroscopic coverage to $R_{200}$, while smaller points are substantially more incomplete. These points indicate that the error introduced by adopting the MaxBCG relation is approximately a factor of 2 . This error estimate is also consistent with an examination of Figure 4 of Becker et al. (2007).

At higher redshifts, three of our clusters have extensive membership information. We estimate that MS0015.9+1609 has $~ 200$ members based on several studies (Dressler \& Gunn 1992; Ellingson et al. 1998) and that MS2053.7-0449 has $~ 100$ members (Tran et al. 2005). Note that these estimates are different from those presented in Eastman et al. (2007) due to updated completeness corrections and the change in the absolute magnitude threshold. For MS 1054-03 we estimate that there are $\sim 300$ members from the extensive spectroscopic work of Tran et al. (2007). These three clusters are also shown in Figure 2 (filled circles). They are consistent with the low-redshift results and a factor of 2 uncertainty in the richness-velocity dispersion relation. While our estimates of the cluster galaxy population for these three clusters, as for the low-redshift clusters, are based on all galaxies rather than just red galaxies, the consistency supports the assumption that the integral of the bright end of the galaxy LF in clusters above an evolving $M_{R}$ threshold scales reasonably well with the cluster velocity dispersion independent of redshift, even if there is evolution in the colors of the cluster galaxies. The number of AGNs, estimate of the inactive population, AGN fraction, and spectroscopic completeness for each cluster is listed in Table 7.

\subsection{Cluster AGN Fraction and Evolution}

The AGN fraction for any single cluster is very small and it is uncertain due to small number statistics. In addition, the AGN fraction may vary from cluster to cluster due to correlations with other cluster properties such as velocity dispersion (Sivakoff et al. 2008). The AGN fraction may also depend on variation in the properties of the galaxy population within each cluster (e.g., mass, SFR, and morphology). We, therefore, have binned the cluster sample in two ways to characterize variations with redshift. First, we simply split the sample at $z=0.4$. This choice is primarily motivated by the transition between where we rely on our own measurements and where we largely rely on other work. It also approximately divides the sample into two (17 clusters at $z<0.4,15$ at $z>0.4$ ). This yields completenesscorrected AGN fractions of $f_{A}(z=0.19)=0.00134_{-0.00087}^{+0.0018}$ and $f_{A}(z=0.72)=0.0100_{-0.0023}^{+0.0029}$, or approximately a factor of 8 increase in the AGN fraction (see Table 8) from a median redshift of 0.19 to a median redshift of 0.72 . AGN fractions without the completeness correction are also listed in Table 8. The uncertainties on these quantities are double-sided, $1 \sigma$ confidence limits (Gehrels 1986). The increase in the AGN fraction is formally significant at the $3.8 \sigma$ level. We also split the sample into three bins with $z<0.3,0.3<z<0.6$, and $z>0.6$ to better resolve the continued increase at high redshift that is apparent in the raw data for individual clusters. This binning yields AGN fractions of $f_{A}(z=0.15)=0.0019_{-0.0012}^{+0.0025}, f_{A}(z=$ $0.45)=0.0031_{-0.0015}^{+0.0024}$, and $f_{A}(z=0.81)=0.0147_{-0.0039}^{+0.0050}$. The measured evolution between the lowest and highest bins is also a factor of 8 and in good agreement with the other binning scheme. We note that the observed evolution is also well fitted by a simple power-law scaling as $f_{A} \propto(1+z)^{\alpha}$ where $\alpha=5.3_{-1.7}^{+1.8}$, although 
the power-law index is strongly correlated with the $z=0$ value of the AGN fraction.

The factor of 8 evolution of the AGN fraction is smaller but consistent with the order of magnitude evolution observed by Eastman et al. (2007). They measured $f_{A}(z=0.2)=$ $0.0007_{-0.0007}^{+0.0021}$ and $f_{A}(z=0.6)=0.020_{-0.008}^{+0.012}$ for $L_{X, H}>$ $10^{43} \mathrm{erg} \mathrm{s}^{-1}$, although for a lower and fixed galaxy absolute magnitude of $M_{R}=-20$. At $z=0$ our galaxy absolute magnitude threshold is approximately a magnitude brighter than that used by Eastman et al. (2007) and the offset increases linearly with redshift. This difference in absolute magnitude threshold can readily account for the change in the lowredshift fraction because most of the AGNs are associated with luminous cluster galaxies, that is, increasing the galaxy luminosity threshold decreases the denominator and does not affect the numerator of the AGN fraction. In addition, we have since identified a second luminous AGN at low redshift (Sivakoff et al. 2008). At high redshift the change in galaxy luminosity threshold is also important, but in addition the cluster sample is more than three times larger than the Eastman et al. (2007) sample. The low-redshift cluster sample has increased by less than a factor of 2 .

One way to characterize the evolution of the cluster AGN fraction relative to the field is to calculate the integral of the field space density $\Phi\left(L_{X, H}>10^{43}\right)$ as a function of redshift. Integration of the luminosity-dependent density evolution model in Ueda et al. (2003) yields a factor of 5 increase between $z=0.8$ and $z=0.2$, which is somewhat less but consistent with the observed evolution of cluster AGNs. However, this is not a fair comparison because the evolution of field AGNs with $\Phi\left(L_{X, H}>10^{43}\right)$ is not normalized by the evolution of all field galaxies brighter than $M_{R}^{*}+1$ and the cluster AGN fraction is.

While there is not a direct measurement of the field AGN fraction similar to our calculation for clusters (although see Lehmer et al. 2007), we can estimate this quantity by dividing the integral of the field hard X-ray LF from Ueda et al. (2003) by the integral of the galaxy LF. We have identified three surveys that report LF measurements for the $R$ band and approximately span the same redshift range of this work. The first of these is the VIMOS-VLT Deep Survey (Ilbert et al. 2005), which is based on $U B V R I$ photometry, $\sim 11,000$ spectra to $I_{A B}=24$ mag and extends from $z=0.05$ to $z=2$ (although their lowest redshift point is taken from SDSS; Blanton et al. 2003). We also show results from two measurements based on photometric redshift data: the Las Campanas Infrared Survey (LCIRS; Chen et al. 2003), which is mostly based on UBVRIH measurements and presents the LF for $z=0.5-1.5$, and the Great Observatories Origins Deep Survey (Dahlen et al. 2005), which is based on $U$ through $K$ observations and presents the galaxy LF to $z=2$. While these photometric redshift surveys may have more systematic uncertainties than the LF based on spectroscopic measurements, they have the virtue that they have measured the luminosity function in the rest-frame $R$ band, rather than relied on assumptions about galaxy spectral energy distributions (SEDs) to calculate $K$-corrections. We have calculated the field AGN fraction for each of these surveys and shown the results in Figure 3 (open symbols). At low redshift, the AGN fraction calculated with the Ilbert et al. (2005) LF is approximately a factor of 5 above the cluster fraction, which is consistent with the difference between the field and clusters seen by Dressler et al. (1999) for spectroscopically identified AGN. At higher redshifts $(z>0.5)$, the field estimates range between a factor of 3 and a factor of 10 above the cluster fraction. These estimates of

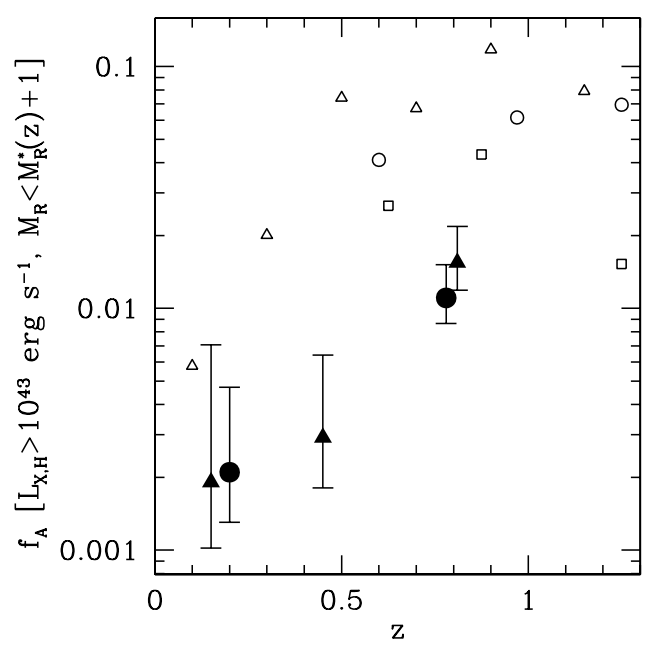

Figure 3. Evolution of the AGN population in clusters from $z=0$ to $z=1.3$ (filled symbols). The fraction of cluster members more luminous than $M_{R}^{*}+1$ with AGNs that have $L_{X, H}>10^{43} \mathrm{erg} \mathrm{s}^{-1}$ is shown in two redshift bins $(z<0.4, z>0.4$; filled circles) and three redshift bins $(z<0.3$, $0.3<z<0.8,0.8<z<1.3$; filled triangles). We also show our estimate of the field AGN fraction based on the galaxy LF estimates by Ilbert et al. (2005, open triangles), Dahlen et al. (2005, open circles), and Chen et al. (2003, open squares). See Section 4.4 for further details.

the field AGN fraction vary so substantially due to the dispersion in estimates of the galaxy LF. In addition, this calculation presupposes that all of the X-ray AGNs are in galaxies more luminous than $M_{R}^{*}(z)+1$. While there is good evidence that most of these luminous X-ray AGN are in relatively luminous galaxies (e.g., Silverman et al. 2009a), there is nevertheless a bias against spectroscopic identification of lower-luminosity $\mathrm{X}$-ray AGN host galaxies. Finally, we note that the relative evolution of galaxies in clusters and the field further complicates this comparison. In future work, we hope to compile sufficient data to calculate the AGN fraction in the field and clusters as a function of galaxy mass. At present the data are insufficient to conclude if the cluster AGN fraction or field AGN fraction evolves more rapidly.

\subsection{Contamination by AGN Associated with Large-Scale Structure}

One concern raised about the physical origin of the ButcherOemler effect is the contribution of projection effects. Diaferio et al. (2001) studied this issue in detail with $N$-body simulations and semianalytic models to distinguish true cluster members from field interlopers that were at the cluster redshift and within the projected $R_{200}$, yet physically outside the cluster $R_{200}$. Diaferio et al. (2001) concluded that up to 50\% of the apparent Butcher-Oemler galaxies at the redshifts of high-redshift clusters may be interlopers. A similar effect may be relevant for the AGN population and such a large contamination would decrease the observed evolution, but not erase it.

While there is no comparable study that directly investigates the projection of AGNs onto high-redshift clusters, there is good evidence that AGNs are associated with the large-scale environment of clusters. Gilmour et al. (2007) identified $11 \mathrm{X}$ ray AGNs (to a lower-luminosity limit of $\sim 10^{41} \mathrm{erg} \mathrm{s}^{-1}$ ) in the A901/2 supercluster at $z \sim 0.17$ and only one was in the densest region of the supercluster. The remainder were mainly in regions of intermediate density. In the vicinity of 3C295 $(z=0.46)$ D'Elia et al. (2008) found evidence for AGNs associated with a filamentary structure. At yet higher redshifts this trend is also 
Table 9

High-Redshift AGNs Associated with Large-Scale Structure around Clusters

\begin{tabular}{|c|c|c|c|c|c|c|c|c|}
\hline $\begin{array}{c}\mathrm{AGN} \\
(1)\end{array}$ & $\begin{array}{c}\text { Cluster } \\
(2)\end{array}$ & $\begin{array}{c}z \\
(3) \\
\end{array}$ & $\begin{array}{c}R \text { (mag) } \\
(4)\end{array}$ & $\begin{array}{c}\log L_{X, H}\left(\mathrm{erg} \mathrm{s}^{-1}\right) \\
(5)\end{array}$ & $\begin{array}{c}\delta v / \sigma\left(\mathrm{km} \mathrm{s}^{-1}\right) \\
(6)\end{array}$ & $\begin{array}{c}\Delta R(\operatorname{arcmin}) \\
(7)\end{array}$ & $\begin{array}{c}R / R_{200} \\
(8)\end{array}$ & $\begin{array}{c}\text { Class } \\
(9) \\
\end{array}$ \\
\hline CXOSEXSI J084846.0+445945 & RX J0848.7+4456 & 0.567 & 21.45 & 43.1 & 1.99 & 3.51 & 1.16 & ELG \\
\hline CXOMP J230300.9+084659 & RXJ2302.8+0844 & 0.738 & 21.71 & 44.23 & 2.81 & 4.46 & 1.2 & BLAGN \\
\hline CXOSEXSI J171807.6+670647 & RXJ1716.4+6708 & 0.797 & 21.75 & 44 & 1.83 & 7.8 & 1.59 & BLAGN \\
\hline CXOU J105710.6-033500 & MS 1054-03 & 0.832 & 21.93 & 43.14 & 1.27 & 4.57 & 1.18 & ALG \\
\hline CXOSEXSI J091040.8+542006 & RDCS J0910+5422 & 1.097 & 22.38 & 43.1 & 2.74 & 2 & 1.13 & ELG \\
\hline CXOSEXSI J084903.9+445023 & LynxE & 1.276 & 23.92 & 43.2 & 2.95 & 1.76 & 1.03 & ELG \\
\hline
\end{tabular}

Notes. AGN associated with large-scale structure around the subset of high-redshift clusters with complete X-ray coverage to twice the projected virial radius. This is the subset of AGNs that satisfy the redshift selection criterion, but have a projected distance of $1<R / R_{200} \leqslant 2$. Columns are identical to Table 2 . The data for CXOU J105710.6-033500 are from van Dokkum et al. (2000) for the redshift, magnitude, and classification and the X-ray data are from Johnson et al. (2003). This sample is described in further detail in Section 4.5.

apparent. Kocevski et al. (2009) found X-ray AGNs associated with the CL1604 supercluster at $z \sim 0.9$, which contains eight confirmed groups and clusters. These AGNs mostly avoid the densest regions of the clusters and are located on the outskirts of the most massive clusters, that is they are associated with poorer clusters and groups.

We examined our data to determine if there were a population of AGN outside the projected $R_{200}$ for these clusters similar to those seen in the two superclusters. This is only possible with the subset of the sample with substantial coverage beyond $R_{200}$. Eight of the clusters have Chandra coverage that extends to $2 R_{200}$. There are six AGNs between $R_{200}$ and $2 R_{200}$ that meet our velocity cuts for cluster membership (Table 9) compared to eight AGNs within $R_{200}$ for these same clusters. The larger number within the clusters suggests the opposite trend from the two supercluster studies described above, although these results are not truly in conflict because the supercluster studies encompassed a much larger area outside of dense clusters than this study. The different large-scale environments associated with these clusters and the superclusters suggest a more quantitative comparison would not be meaningful. These large-scale structure data also provide a crude means to estimate the likelihood of chance juxtapositions of AGNs associated with large-scale structures onto the clusters. If interloper AGNs have the same surface density within $R_{200}$ as between $R_{200}$ and $2 R_{200}$, then the six we identified in an area of $3 \pi R_{200}$ suggest we should expect at most two interlopers compared to the eight AGNs we see within $R_{200}$. This line of argument suggests that the interloper fraction is $25 \%$, which is small compared to the observed evolution signature.

\subsection{Uncertainties}

One major potential source of systematic error is the use of the MaxBCG richness estimator to estimate the fraction of cluster galaxies more luminous than $M_{R}^{*}+1$. In Section 4.3, we estimated that there is a factor of 2 uncertainty in the use of this relation. This uncertainty is mainly important for the high-redshift subsamples as the low-redshift subsamples have more complete spectroscopic membership data. If we randomly introduce a factor of 2 uncertainty in each cluster, the effect is negligible when averaged over the 15 clusters with $z>0.4$ compared to the factor of 8 evolution in the AGN fraction.

As mentioned previously, another valid concern with the MaxBCG estimator is that it is calibrated to the number of red galaxies in the cluster and this population may not all be in place at $z=0.4$ and higher. For our application it does not matter if the galaxies are red or not, just that they are in the cluster. Furthermore, if we have overestimated the number of galaxies brighter than $M_{R}^{*}+1$ then we have underestimated the evolution of the AGN fraction and our result is yet more statistically significant. The assumption that all of the galaxies are red does impact the $K$-correction we use to estimate the spectroscopic limit corresponding to $M_{R}^{*}(z)+1$ and thus the size of our completeness correction. If the galaxies are redder, then the $K$-correction would be smaller, the apparent magnitude limit would be brighter, and the completeness correction would be smaller. The implication would be that we have preferentially overestimated the AGN fraction at high redshifts because completeness corrections are only applied to the high-redshift clusters. While the average completeness correction approaches $25 \%$ (see Table 8 ), in practice the spectroscopic completeness is not a strong function of apparent magnitude (e.g., see Section 4.2, Silverman et al. 2005; Eckart et al. 2006) and we consequently expect much less than a $25 \%$ reduction in the evolution. The evolution of the host-galaxy population is also important because if there were less fading of $M^{*}(z)$ than we assume, then the completeness limit would be too bright and we would have underestimated the AGN fraction at high redshift.

The value of the cluster velocity dispersion introduces additional uncertainty to this calculation in two ways. First, many of the direct measurements of the cluster velocity dispersion, particularly for high-redshift clusters, are based on small samples of galaxies and thus the velocity dispersion itself may be uncertain, particularly if the galaxy velocity distribution is not Gaussian. Second, as noted above, the cluster velocity dispersion has not been directly measured for several clusters and we instead used the X-ray temperature and the results of Xue \& Wu (2000) to estimate the velocity dispersion and this has a $30 \%$ scatter. We checked both of these concerns with a measurement of the scatter between $\sigma$ and $T_{X}$ for the 10 high-redshift clusters with measurements of both quantities and the mean deviation is $\sim 220 \mathrm{~km} \mathrm{~s}^{-1}$ if we exclude 3 C295, which has a substantially higher velocity dispersion (1642 $\mathrm{km} \mathrm{s}^{-1}$, Girardi \& Mezzetti 2001) than expected from its X-ray temperature (5.3 K from Vikhlinin et al. 2002). This mean deviation corresponds to approximately a factor of 2 uncertainty in the richness, which is comparable to the uncertainty we derived for the richness estimator. From this analysis we similarly conclude that this source of uncertainty does not substantially affect our results.

A related evolutionary effect is that the velocity distributions of the high-redshift clusters may be systematically more nonGaussian than low-redshift clusters because the high-redshift clusters are less likely to be relaxed. If the cluster velocity dispersion were overestimated, then the richness and $R_{200}$ would 


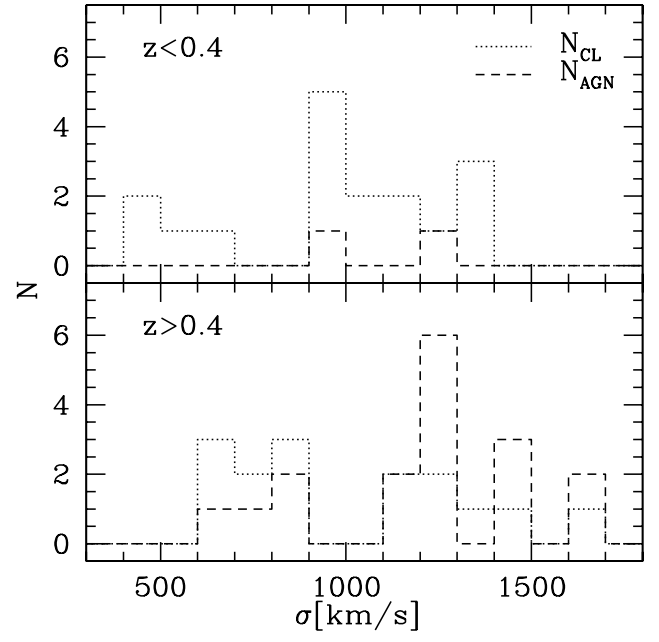

Figure 4. Histograms of the number of clusters with a given velocity dispersion (dotted line) and the number of AGNs in clusters of a given velocity dispersion (dashed line) for the low-redshift $(z<0.4$; top panel) and the high-redshift $(z>0.4$; bottom panel) subsamples. The cluster samples are reasonably well matched within these two redshift bins.

be overestimated as well. This, in turn, would lead to an underestimate of the AGN fraction in high-redshift clusters. Jeltema et al. (2005) measured power ratios from Chandra observations of the IGM for a large sample of clusters out to $z \sim 1$ and found good evidence that high-redshift clusters are less relaxed than low-redshift clusters, so this potential source of systematic error would lead us to underestimate the AGN fraction. Nine of our clusters were analyzed in the Jeltema et al. (2005) study, including eight in our high-redshift sample. We compared the AGN fractions and the power ratios for these clusters, but did not find a significant trend. Unfortunately we do not have sufficient redshift data for most high-redshift clusters to look for non-Gaussianity in the galaxy velocity distribution, although note there is no evidence for a trend between dynamically disturbed clusters and AGN fraction at low redshift (Martini et al. 2007).

Finally, we consider the evolution of the cluster population to determine if the higher-redshift clusters represent the progenitor population of the lower redshift clusters. As noted previously, observations at low redshift indicate that the AGN fraction depends on environment and specifically that the AGN fraction is higher in lower velocity dispersion environments (Sivakoff et al. 2008; Arnold et al. 2009). Therefore, if our high-redshift clusters are the progenitors of lower velocity dispersion clusters or massive groups, then the observed evolution may not be as significant. As many of the high-redshift clusters are $\mathrm{X}$ ray selected, they are generally high-mass clusters and are reasonably well matched to the lower-redshift sample (see Figure 4 and Table 7). Following Finn et al. (2005) and Poggianti et al. (2006), we have estimated the velocity dispersions of the progenitors of the high-redshift cluster sample and find they are in good agreement. For example, the progenitor of a $1000 \mathrm{~km} \mathrm{~s}^{-1}$ cluster at $z=0$ has $800 \mathrm{~km} \mathrm{~s}^{-1}$ at $z=0.6$ (Poggianti et al. 2006), or only about $100 \mathrm{~km} \mathrm{~s}^{-1}$ less than the difference between our low-redshift and high-redshift subsamples. The sense of this trend is that the high-redshift sample is actually somewhat more massive than the typical progenitor of the low-redshift sample and therefore the minor mismatch in cluster masses is more likely to have dampened rather than enhanced the measured evolution of the AGN fraction.

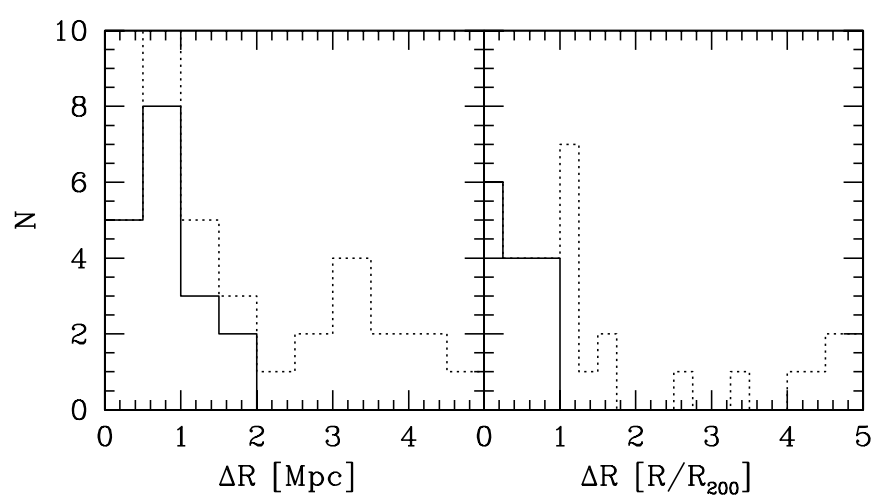

Figure 5. Histograms of the AGN clustercentric distances in terms of Mpc (left) and normalized to $R_{200}$ (right) for cluster AGN with $z>0.4$. The distribution of the confirmed cluster members (solid line) is much more centrally peaked when expressed in terms of Mpc than in terms of $r / R_{200}$. Other AGNs associated with large-scale structure (with $R>R_{200}$ ) are also shown (dotted line).

\section{PROPERTIES OF THE CLUSTER AGN}

\subsection{Distribution}

The projected radial and velocity distributions of the AGNs provide valuable additional information about the origin of the AGNs. For example, if the AGNs are preferentially located in the cluster outskirts, or preferentially have a higher velocity dispersion than the cluster mean, this may indicate that their host galaxies have relatively recently entered the cluster potential. This is known to be the case for emission-line galaxies (Biviano et al. 1997; Dressler et al. 1999). At low-redshifts and for lower-luminosity X-ray AGNs, Martini et al. (2007) found that $L_{X}>10^{42} \mathrm{erg} \mathrm{s}^{-1}[0.5-8 \mathrm{keV}]$ AGNs were more centrally concentrated than typical cluster galaxies, while AGNs an order of magnitude less luminous had the same distribution as the inactive galaxy population. For both luminosity thresholds, the velocity distribution of the AGNs was consistent with the galaxy population.

It is more challenging to compare these higher-luminosity $\mathrm{X}$-ray AGNs to the host galaxy population because we lack membership data for nearly all of the high-redshift clusters. Nevertheless, we can compare the distribution of sources to the typical distribution of cluster galaxies and to the excess surface density distribution found by surveys of X-ray point sources toward distant clusters. In Figure 5, we present a histogram of the number of X-ray AGNs from the cluster center as a function of distance in both physical units (Mpc) and normalized to $R_{200}$. While the sample is small, two results are apparent from the figure. First, there are approximately equal numbers of AGNs outside $0.5 R_{200}$ as inside it, whereas if the AGNs traced the cluster galaxy distribution we would expect them to be more centrally concentrated. Second, the radial distribution is more strongly peaked when plotted in physical units than normalized to $R_{200}$.

While we do not have detailed information on the radial distribution of the cluster galaxy populations in these clusters, we do have extensive data on nearby clusters from Christlein $\&$ Zabludoff (2003). For these clusters we have investigated the cluster galaxy distribution with the same selection criteria $\left(R<R_{200}, M_{R}<M_{R}^{*}+1, \Delta v<3 \sigma\right)$ and find that $70 \%$ of the galaxies fall within $0.5 R_{200}$, whereas 10 of 18 luminous AGNs at $z>0.4$ are within $0.5 R_{200}$. The binomial probability is only $14 \%$ that we would find 10 or fewer AGNs within $0.5 R_{200}$ if we expected $70 \%$. There is, thus, a mild tendency 


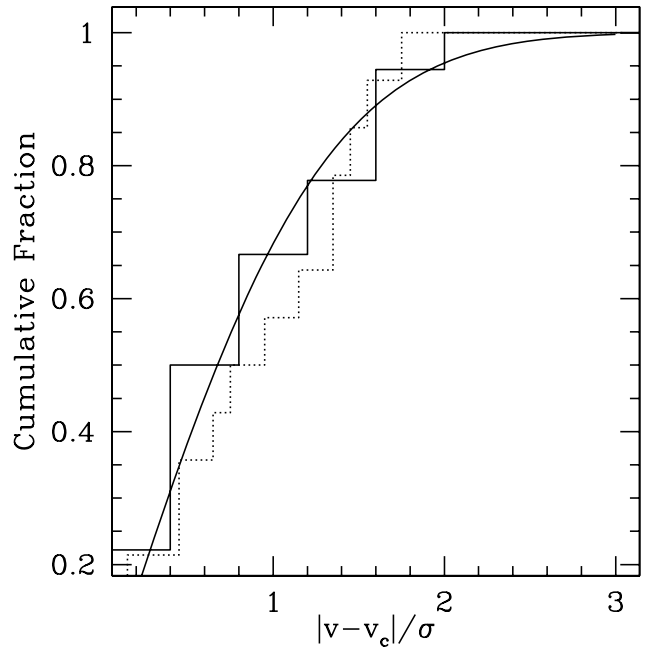

Figure 6. Histogram of the cumulative velocity distribution of cluster AGNs normalized to the cluster velocity dispersion for the 18 cluster AGNs with $z>0.4$ (solid histogram). The AGN velocity distribution is consistent with a Gaussian distribution (solid curve) and the $L_{X, B} \geqslant 10^{42} \mathrm{erg} \mathrm{s}^{-1}$ AGNs from Martini et al. (2007, dotted histogram).

for luminous AGNs to be distributed toward the outskirts of the clusters, although this does make the substantial assumption that the radial distribution of galaxies within clusters is similar at $z \sim 0.8$ and the present. This broad distribution in radius is in contrast to our earlier results on lower-luminosity AGNs in lower-redshift clusters. At low redshift we found that $50 \%$ of the luminous AGNs were within $0.1 R_{200}$ (Martini et al. 2007). Better statistics could determine if the AGNs are preferentially located in the outskirts of clusters compared to all cluster galaxies. That would be consistent with the hypothesis that AGNs are triggered by mergers during infall. From simulations, Ghigna et al. (1998) found that mergers between galaxies do not occur within the virial radius. We note that Berrier et al. (2009) simulated the formation of 53 galaxy clusters and find most cluster galaxies do not experience "preprocessing" in group environments and therefore processes specific to clusters must largely be responsible for the differences between cluster and field galaxies.

The second result has interesting implications for studies that use the surface density distribution of excess sources to characterize the distribution of AGNs in clusters (Ruderman \& Ebeling 2005; Gilmour et al. 2009; Galametz et al. 2009). These studies generally plot the excess surface density as a function of physical distance from the cluster center and find a central peak in surface density. Our results indicate that the true distribution may be flatter than implied by use of the physical (proper) distance from the cluster core. This is because those surveys, like the present study, include clusters with a wide range of masses and consequently a wide range of $R_{200}$. Simply adding the distributions for all clusters without renormalizing each observation for the size of the cluster will produce an artificial central peak due to the mass range of the cluster sample.

If the cluster AGNs are associated with a population that recently entered the cluster potential, the host galaxies may also be preferentially on more radial orbits and have a larger velocity dispersion than that of all cluster galaxies. As noted previously, this is true of the emission-line galaxy population in clusters. In Figure 6, we plot the cumulative velocity distribution for all 18 AGNs with $z>0.4$ normalized by the cluster velocity dispersion. The distribution is in excellent agreement with a Gaussian distribution and we therefore find no evidence that the cluster AGNs have a larger velocity distribution that would be consistent with more radial orbits. This was also found for the 14 relatively luminous $\left(L_{X, B} \geqslant 10^{42} \mathrm{erg} \mathrm{s}^{-1}\right)$ AGNs studied by Martini et al. (2007). A better test would be to compare the AGN host population to the absorption-line galaxies in the clusters since the velocity dispersion estimates for many of these clusters may be biased toward the emission-line galaxy population because it is easier to measure redshifts for them. While this is not the case for those whose velocity dispersions are estimated from X-ray data, it may also be true of the calibration sample for the relations between X-ray properties and galaxy velocity dispersion.

\subsection{Luminosity Function}

We have begun to acquire sufficient numbers of cluster AGNs that it is possible to compare the X-ray luminosity function (XLF) between clusters and the field, as well as the cluster XLF at different redshifts. A comparison between the cluster and field XLF is interesting because differences between the two would be a signature of environment-dependent downsizing. There is evidence that this is true of star formation in different environments. For example Kauffmann et al. (2004) found that substantial star formation is only present in higher-mass galaxies in lower density environments in the local universe. If the cluster black holes primarily grew at higher redshifts than field black holes, similar to the earlier formation epoch expected for the stellar populations in luminous cluster galaxies, then the cluster LF at high-redshift may have a similar shape to the present-day XLF in the field. One test of this hypothesis is to compare the characteristic luminosity $L_{X}^{*}$ between clusters and the field. If the cluster AGNs primarily grew at an earlier epoch, $L_{X}^{*}$ would be smaller in clusters relative to the field at a given redshift.

It is reasonable to compare the shape of the XLF between clusters and the field because the XLF is a measurement of the X-ray sources alone within well defined volumes, although the caveats associated with large-scale structure discussed in Section 4.5 do apply. This is different from the case in Section 4.4, where we noted that the comparison of the evolution of the AGN fraction and the integrated space density was not comparing identical quantities because the AGN fraction includes information about the galaxy population. The one assumption that we do make is that all of the X-ray sources are hosted by galaxies above our threshold, but this is reasonable given Figure 1. In addition, the normalization remains arbitrary because it is challenging to define a total volume for the cluster AGN sample, although this is not necessary because the shape of the XLF already provides useful information. In Figure 7, we plot the cluster XLF for our $z>0.4$ sample compared to the field XLF at the median cluster redshift of $z=0.8$ from Ueda et al. (2003). The cluster XLF is in reasonable agreement with the field XLF at the same redshift, although the statistics are quite limited. As motivation for future work, we also plot the field XLF at lower redshift $(z=0.1$, dotted line). For the lowerredshift XLF, $L_{X}^{*}$ is smaller and consequently all $L_{X, H} \geqslant 10^{43}$ AGNs are above the characteristic luminosity, while these data straddle $L_{X}^{*}$ in the field XLF at $z=0.8$. Improved statistics for cluster X-ray AGNs at $z>0.4$ could determine if there is also a break in the cluster XLF, or if it is more similar to the field $\mathrm{XLF}$ at lower redshift.

The evolution of the cluster XLF with redshift is also relevant for the origin of X-ray AGNs in lower-redshift clusters. If cluster 


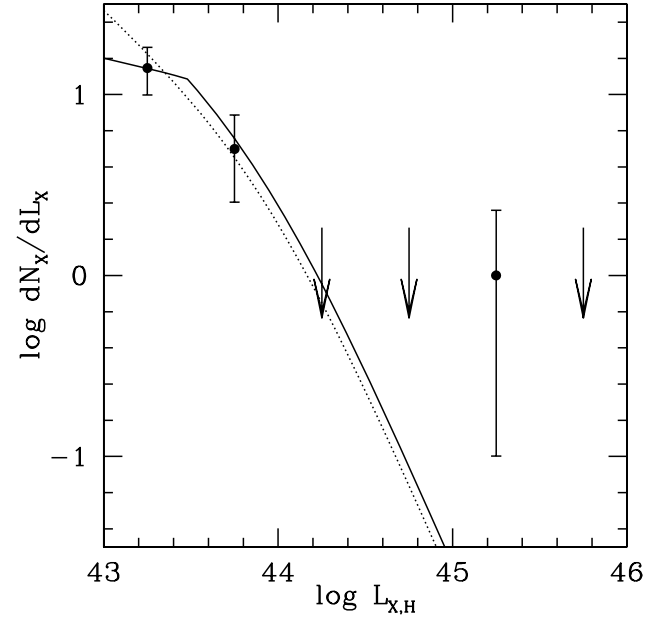

Figure 7. Hard X-ray LF of cluster AGNs at $z>0.4$ compared to the field XLF from Ueda et al. (2003) at the median cluster redshift $(z=0.8$, solid curve) and at low redshift ( $z=0.1$, dotted curve). The field XLFs have been renormalized to be consistent with the cluster measurements in the first two luminosity bins. The arrows are upper limits calculated with Poisson statistics.

AGNs at the present day are simply the descendants of AGNs at higher redshift that have been fading for several Gyr, then the difference between the low-redshift and high-redshift cluster XLF should be consistent with pure luminosity evolution. In contrast, if there is substantial retriggering of low-luminosity AGNs in low-redshift clusters, or if other mechanisms are capable of fueling AGNs in clusters, then the cluster XLF evolution may not be consistent with just luminosity evolution. A signature of other fueling or triggering mechanisms would be a substantially larger population of lower-luminosity AGNs in present-day clusters compared to expectations from the highredshift population. While pure luminosity evolution would be surprising because this is not observed in field AGNs, the most luminous cluster galaxies are consistent with passive evolution. Better measurements of the cluster XLF over a broader range in luminosity could investigate this hypothesis.

\subsection{Host-Galaxy Properties}

Both the colors and morphologies of low-luminosity $\left(\sim 10^{41}\right.$ $\mathrm{erg} \mathrm{s}^{-1}$ ) AGNs in low-redshift clusters suggest they are primarily hosted by galaxies dominated by light from their old stellar populations (Martini et al. 2006). This becomes progressively less true for higher-luminosity AGNs and ground-based observations of the most luminous sources $\left(\geqslant 10^{43} \mathrm{erg} \mathrm{s}^{-1}\right)$ in Abell 2104 (Martini et al. 2002) and Abell 754 (Arnold et al. 2009) indicate that they have late-type morphologies, although their hosts are luminous. In addition, these more luminous AGNs are more likely to exhibit visible-wavelength AGN spectral signatures than their lower-luminosity counterparts.

While the spectroscopic classification of the high-redshift sample is fairly subjective because of variations in wavelength coverage and signal-to-noise ratio, the spectroscopic classifications reported by Silverman et al. (2005) and Eckart et al. (2006) support the low-redshift results. They classified six of $17 \mathrm{X}$-ray AGNs as BLAGN, 9 as other emission-line galaxies, and the remaining two as absorption-line galaxies. The vast majority of the higher-luminosity AGNs have substantial line emission, even with the bias against redshift measurements for sources without strong emission lines. We note for comparison that two of the six AGNs in the large-scale structure sample are classified as BLAGN and the other four are evenly split between emission-line and absorption-line galaxies. These other sources are thus similar to the cluster AGNs.

Several of the high-redshift clusters also have HST observations suitable to study the morphologies of the cluster galaxies. The largest survey of X-ray source morphology in high-redshift clusters is that by Martel et al. (2007), who investigated the fields of five high-redshift clusters: RX J0152-1357, RX J0849+4452, RDCS J0910+5422, MS 1054-0321, and RDCS J1252-2927, and the middle three clusters overlap this sample. For the entire field sample they classify half of the X-ray counterparts as early-type, $35 \%$ as late-type, and $15 \%$ as irregular galaxies. For the six cluster members in their sample, they found half are in early-type hosts, two in late-type hosts, and one in an irregular galaxy. In addition, three of these cluster AGN hosts are in interacting systems. The specific overlaps with this sample are CXOU J091043.3+542152 and CXOMP J105650.6-033508 and both have early-type morphologies (their other member in RDCS J0910+5422 falls slightly below our luminosity threshold).

\subsection{Implications for the Sunyaev-Zel'dovich Effect}

Many cluster surveys are currently planned or in progress that use the Sunyaev-Zel'dovich effect to identify large numbers of clusters (e.g., Kosowsky 2003; Ruhl et al. 2004). This effect is caused by inverse Compton scattering of Cosmic Microwave Background (CMB) photons off hot electrons in the ICM that changes the spectrum of the CMB in the direction of a cluster (e.g., Carlstrom et al. 2002). The main virtue of this effect is that it is redshift independent, and consequently can be used to detect (the hot electrons associated with) clusters out to high redshifts. However, mechanical heating by AGNs in the cluster may contribute to the thermal energy of the ICM (e.g., Bîrzan et al. 2004) and thus make it more difficult to identify some clusters. Any increase in the AGN population with redshift will also introduce a systematic effect with redshift.

The potential impact of AGNs on SZE cluster surveys was recently examined in detail by Lin \& Mohr (2007). They measured the radio LF in nearby clusters at $1.4 \mathrm{GHz}$ and used measurements of AGNs at higher frequencies (Cooray et al. 1998; Coble et al. 2007) to estimate that on order $10 \%$ of clusters will have an AGN flux comparable to the SZE flux. As a worst-case scenario, they adopted an evolution model where the fraction of radio AGNs increases as $(1+z)^{2.5}$. This model was largely motivated by observations of the radio galaxy LF, which suggested evidence for an increase (Best et al. 2002; Branchesi et al. 2006). If this population evolves at a comparable rate more consistent with the $(1+z)^{5.3}$ rate we observe for luminous X-ray AGNs, then the fraction of substantially contaminated clusters will be higher than predicted by Lin \& Mohr (2007).

\section{DISCUSSION}

The extent of the correlation between the evolution of star formation and AGNs in clusters could provide valuable new insights into how closely related these two processes are. The original work by Butcher \& Oemler $(1978,1984)$ on the evolution of the fraction of blue galaxies in clusters provides a useful first point of comparison to the AGN fraction evolution, in part because we adopted many elements of their methodology. Specifically, Butcher \& Oemler (1984) characterized cluster galaxy evolution with: (1) a fixed criterion to define the sample of interest (a galaxy was classified as blue if the rest-frame $B-V$ color was at least 0.2 mag bluer than the relation exhibited by the 
red galaxies); (2) measurement of this population relative only to cluster galaxies above some luminosity threshold $\left(M_{V}=-20\right)$; (3) use of an aperture scaled to the physical properties of individual clusters (a circle that contained the inner $30 \%$ of the cluster galaxy population). With these definitions, Butcher $\&$ Oemler (1984) found that the blue galaxy fraction increased from $f_{B} \sim 0.03$ at $z \leqslant 0.1$ to $f_{B} \sim 0.25$ at $z=0.5$ for relatively compact, concentrated clusters, or approximately an order of magnitude.

One of the most recent and comprehensive studies of the evolution of star formation in clusters is the work of Poggianti et al. (2006). These authors used the [O II] 33727 line as a tracer of star formation, rather than color, and measured the fraction of galaxies with [O II] emission (equivalent width $>3 \AA$ ) as a function of both cluster redshift and cluster velocity dispersion. Their sample includes 25 clusters with $z=0.4-0.8$ and another 10 groups in the same redshift range, while they have a large local comparison sample at $z=0.04-0.08$ from the SDSS. They measure the $\left[\mathrm{O}_{\mathrm{II}}\right]$ fraction $f_{[\mathrm{O}}$ II] relative to an evolving absolute magnitude limit $M_{V, \text { lim }}$ that varies from -20.5 at $z=0.8$ to -20.1 at $z=0.4$, while the local limit was $M_{V}<-19.8$. Their main results are that there is substantial evolution in $f_{[\mathrm{O}}$ II] and that there is substantial variation in $f_{[\mathrm{OII}]}$ with velocity dispersion at a given redshift. Given the velocity dispersion dependence, a direct comparison of the evolution of $f_{[\mathrm{O} \text { II] }}$ with $f_{A}$ is not meaningful for different cluster samples. Instead, we have used their upper envelope for $\left.f_{[\mathrm{O}}(\sigma]\right)$ at high redshift and their envelope prescription at low redshift to estimate $f_{[\mathrm{O} \text { II] }}$ for each of our clusters and then computed the average $f_{[\mathrm{O} \text { II] }}$ for each of the subsamples shown in Table 8. These relations predict an increase in $f_{[\mathrm{O}}$ II] of less than a factor of 2 from the low-redshift to the high-redshift subsamples, or substantially less than the factor of 8 we observe for the AGN fraction.

These results are interesting, although numerous caveats forestall too much interpretation of the relative rates of evolution. One major concern is that there is likely downsizing in clusters similar to what is observed in the field (e.g., Cowie et al. 1996; Hasinger et al. 2005; Silverman et al. 2008; Yencho et al. 2009), that is, the relative number of galaxies with star formation or AGNs activity above a certain threshold varies with redshift. The direct implication of this for the AGN fraction is that the evolution of the AGN fraction over a given redshift range is expected to depend on luminosity, just as the rate of evolution of the AGN space density is observed to vary in the field as a function of minimum luminosity (e.g., Ueda et al. 2003). This is similarly a complication for interpretation of the evolution of star formation, and consequently limits direct comparison of the mere rates of evolution of star formation and AGNs above some threshold. For example, while Poggianti et al. (2006) have similarly used an evolving galaxy luminosity threshold to characterize the evolution of the star-forming galaxy fraction, their galaxy luminosity threshold is over a magnitude fainter and therefore they have measured the evolution in a population that includes many more fainter cluster members. However, these concerns are not an obvious limitation to comparisons that use the same luminosity threshold to separately compare either AGNs or star formation across different environments, particularly when the evolution of the star formation rate and AGN luminosity is tied to the same galaxy population. For example, if the relative rates of evolution of AGNs and star formation in $<M_{R}^{*}+1$ galaxies were different in the field and clusters, this would suggest a limit to the extent of the apparent coevolution of black holes and galaxies in at least one of these environments.
Another concern about a direct comparison to these measurements of the evolution of the star-forming galaxy population is that [O II] emission is more susceptible to reddening and metallicity effects relative to other star formation indicators, such as $\mathrm{H} \alpha$ (Kewley et al. 2004). Many ISO studies (summarized by Metcalfe et al. 2005) found evidence for an increase in star formation in clusters at higher redshifts, and that the increase appeared to be greater than that predicted by UV continuum or visible-wavelength spectroscopic diagnostics. Spitzer observations of clusters have also found substantial, often obscured, star formation in high-redshift clusters (Geach et al. 2006; Marcillac et al. 2007; Bai et al. 2007). Geach et al. (2006) used new Spitzer data for two clusters and data for five others from the literature to estimate the star formation rate normalized by the cluster mass. They found evidence for an increase in higher-redshift clusters, but also substantial variation between clusters at the same redshift. Saintonge et al. (2008) used a larger sample of eight clusters with $24 \mu \mathrm{m}$ Spitzer data to study the evolution of the fraction of obscured star-forming galaxies from $z=0.02-0.83$. They find that the fraction of cluster galaxies with star formation rates above $5 M_{\odot} \mathrm{yr}^{-1}$ increases from $3 \%$ at $z=0.02$ to $13 \%$ at $z=0.83$ and that this is stronger evolution than exhibited by color selection, such as the criteria of Butcher \& Oemler (1978, 1984). The star-forming galaxies they identify in these clusters are also mostly disjoint from the Butcher-Oemler galaxies and consequently when they sum the blue and mid-infrared galaxies the fraction of star-forming galaxies increases to $\sim 23 \%$ at high redshift.

Several of these Spitzer studies overlap clusters that are also in our sample and it is interesting to see if there is a direct correspondence between the AGNs and mid-infrared sources detected by Spitzer. The massive cluster MS1054-03 was studied by Bai et al. (2007) and their $24 \mu \mathrm{m}$ sources include the two X-ray AGNs identified by Johnson et al. (2003). Saintonge et al. (2008) have three clusters in common with our sample: MS0451.6-0305, MS2053.7-0449, and MS 1054-03, although they do not provide information on individual sources. While not in our sample, the study of RX J0152.7-1357 $(z=0.831)$ by Marcillac et al. (2007) found that the two most luminous $24 \mu \mathrm{m}$ sources (of 22 confirmed members) were also X-ray AGNs. Similarly, Geach et al. (2009) found that one (of 12) of the luminous infrared galaxies $\left(L_{I R}>10^{11} L_{\odot}\right)$ in CL0024+16 $(z=0.4)$ was obviously an AGN based on their infrared data alone. At lower redshifts, Gallagher et al. (2008) have also used Spitzer data to identify AGNs and star-forming galaxies in Hickson Compact Groups.

Saintonge et al. (2008) explored whether or not the increase in the fraction of obscured star formation in high-redshift clusters is related to infall. They speculate that the increase in star formation reflects the infall of new members and note that most of the MIPS-detected cluster galaxies are not projected onto the cluster core (inner 500pc). Over larger scales, the work of Gallazzi et al. (2009) explored the obscured star formation fraction as a function of environment in the Abell 901/902 supercluster at $z=0.165$. They found more obscured star formation at intermediate densities than in the cluster cores, similar to the distribution of the AGN population studied by Gilmour et al. (2007) in the same supercluster. If there is a substantial increase in the obscured star formation fraction in the intermediate densities around clusters, and the star formation in this environment increases with redshift, then projection of some of these structures onto the cluster core may contaminate the cluster estimates. 
As discussed in Section 4.5, AGNs in the large-scale environments around massive clusters may also project onto cluster cores. To better evaluate this possibility, it is useful to both directly measure the AGN population immediately outside clusters and measure the AGN population in intermediate densities more generally. Just as Poggianti et al. (2006) found that the fraction of [O II]-emitting galaxies increases in lower velocity dispersion environments, the AGN fraction as a function of environment is important because the environmental dependence may provide new information on the processes that drive AGN evolution. Both the XMM-Newton observations of the COSMOS fields (Silverman et al. 2009a, 2009b) and Chandra observations of the Extended Growth Strip from DEEP2 (the All-wavelength Extended Groth strip International Survey, AEGIS; Georgakakis et al. 2008a; Georgakakis et al. 2008b) have estimated the AGN fraction in groups of galaxies or as a function of local overdensity at high redshifts. Georgakakis et al. (2008b) found that Xray AGNs are more frequently found in groups than in the field, which they connect to their observation that the X-ray AGN host galaxies are often red, luminous galaxies that tend to reside in denser environments, although they also found that this trend may reverse for the most powerful AGN. In a narrower redshift range from $0.7<z<0.9$ and for $M_{B}<-20$ mag they found that the AGN fraction is comparable in groups and the field and about $5 \%$. This is approximately a factor of 5 higher than we find in clusters at similar redshifts, although these values are not exactly comparable as the Georgakakis et al. (2008b) AGNs include somewhat lower-luminosity sources than our sample and the host galaxy magnitude limits are somewhat different. Silverman et al. (2009a) also investigated the environment dependence of X-ray AGN hosted by galaxies above a fixed stellar mass and found no strong preference between the field and groups except for the most massive galaxies, while Jeltema et al. (2007) found that the fraction of $[\mathrm{O}$ II]-emitting galaxies in intermediateredshift, X-ray-selected groups $(0.2<z<0.6)$ is similar to clusters at the same redshift.

The clustering analysis by Coil et al. (2009) on the AEGIS data also helps to elucidate the distribution of AGNs at high redshift as a function of environment, AGN luminosity, and host-galaxy mass. They found that the X-ray AGNs have similar clustering to luminous red galaxies and are more likely to reside in groups, while UV-bright QSOs are less strongly clustered and more similar to the field blue galaxy population. This is also similar to the results from Kauffmann et al. (2004) at low redshifts from SDSS, who found that galaxies at a fixed stellar mass that host luminous [O III] emission are twice as common in low-density regions as high. Taken together, the AEGIS and COSMOS results illustrate that the measured AGN fraction depends on both the stellar mass (or luminosity) of the galaxy population and the star formation rate of the host, in addition to the AGN luminosity. This makes a direct comparison between these two surveys, as well as to our work on high-redshift clusters, somewhat problematic. The X-ray range considered by Silverman et al. (2009a) extends over $42<\log L_{0.5-10 \mathrm{keV}}<43.7$, or approximately half an order of magnitude below our X-ray threshold for a typical AGN SED. The X-ray AGNs studied by Coil et al. (2009) extend an order of magnitude fainter than our work to a hardband limit of $L_{X, H}>10^{42} \mathrm{erg} \mathrm{s}^{-1}$. Both of these surveys are therefore dominated by intrinsically less luminous objects. The galaxy mass and luminosity ranges are similarly not identical. In future work, we hope to put all of these high-redshift measurements on an equal basis for a more direct comparison.
While none of these results suggest that there are more luminous AGNs in clusters than groups or the fields out to $z \sim 1$, such a trend may be seen at yet higher redshifts. Observations of cluster galaxies, particularly massive cluster ellipticals, suggest that most of their stars formed earlier than field galaxies (by 0.4 Gyr; van Dokkum \& van der Marel 2007). If the central black holes of these galaxies grew contemporaneously, then perhaps by $z \sim 2$ the AGN fraction will be higher in denser environments. Some interesting support for this picture comes from Chandra observations of the SSA22 protocluster at $z=3.09$ (Lehmer et al. 2009). They found a slightly higher AGN fraction in Lyman Break and Ly $\alpha$-emitting galaxies in the protocluster compared to the field. While this is just one region, observations of the AGN fraction in clusters relative to the field at $z \sim 2$ and above could provide interesting new insights into the coevolution of black holes and galaxies.

\section{SUMMARY}

We have conducted an expanded survey to identify luminous $L_{X, H} \geqslant 10^{43} \mathrm{erg} \mathrm{s}^{-1}$ AGNs in clusters of galaxies from $z \sim 0.05$ to $z \sim 1.3$. At low redshifts we have presented a new X-ray analysis of archival Chandra observations and spectroscopic follow-up of AGN candidates in six new clusters. There are no new, luminous AGNs in these clusters and there are a total of just two luminous AGNs in our sample of 17 clusters with $z<0.4$. These measurements further strengthen the evidence for a very small luminous AGN fraction in low-redshift clusters. An important virtue of the new clusters is that the X-ray and spectroscopic coverage extends to the projected $R_{200}$ radius and therefore they are better matched to observations of highredshift clusters. At higher redshifts we have combined our previous work with literature data on X-ray sources, primarily from the ChaMP and SEXSI surveys, to compile a total sample of 15 clusters at $z>0.4$. In spite of somewhat incomplete spectroscopic coverage of the X-ray sources in these fields, there are 18 luminous AGNs in these clusters.

We parameterize the evolution of the AGN population in clusters in terms of the fraction of luminous galaxies that host AGNs above our luminosity threshold. We have used a variety of techniques to estimate the number of luminous galaxies, defined to have $M_{R}<M_{R}^{*}+1$, in these clusters and calculated the average cluster AGN fraction in several redshift bins. As the low and high-redshift clusters are reasonably well matched in terms of cluster velocity dispersion and X-ray temperature, the increase in the number of AGNs is closely related to the increase in the fraction of galaxies more luminous than $M_{R}^{*}+1$. Specifically, we find that the AGN fraction increases by approximately a factor of 8 from $z \sim 0.2$ to $z \sim 1$. This corresponds to an increase in the AGN population that scales as $(1+z)^{5.3}$. If the radio AGN population in clusters increases by a comparable amount, radio AGNs may impact the identification of clusters as a function of redshift in current and planned SZ surveys. The substantial evolution in the cluster AGN population is also correlated with the evolution of the fraction of star-forming galaxies in clusters known as the ButcherOemler effect. Detailed studies of star formation and AGNs in individual clusters could better quantify the extent that these two phenomena are coupled in clusters or perhaps even individual galaxies. We have also estimated the evolution of the field AGN fraction to compare it to the cluster AGN fraction. While the field AGN fraction is higher at all redshifts, the present data do not suffice to conclude if the rate of evolution is faster or slower 
in clusters. Future measurements of the relative evolution of star formation and black hole growth in clusters and the field could be an important probe of the coevolution of black holes and their host galaxies.

Measurements of the radial distribution of the cluster AGNs provide new information on the origin of AGNs within clusters. Unlike we found in previous work at low redshifts, the AGNs in these high-redshift clusters are not strongly centrally concentrated when their distribution is plotted normalized to the $R_{200}$ radius. This demonstrates that there are substantial numbers in the outskirts of clusters and supports the hypothesis that some cluster AGNs are hosted by relatively gas-rich galaxies that have recently entered the cluster potential. While this excess is not apparent in the velocity distribution, this may be due to biases in the measurement of the cluster velocity dispersion or simply small number statistics. We have also presented the first measurement of the XLF of cluster AGNs at high-redshift and found that it is consistent with the field XLF at the same redshift. This comparison illustrates the future potential of XLF measurements in clusters to measure environment-dependent downsizing in clusters, as well as how the evolution of the cluster XLF can be used to constrain the evolution of black hole growth in clusters.

We are grateful to John Silverman and the referee for many suggestions that have improved this paper. We also acknowledge helpful discussions with Dan Stern and Tommaso Treu. Support for this work was provided by the National Aeronautics and Space Administration through Chandra Award Number AR89014X issued by the Chandra X-ray Observatory Center, which is operated by the Smithsonian Astrophysical Observatory for and on behalf of the National Aeronautics Space Administration under contract NAS8-03060. P.M. is grateful for support from NSF via award AST-0705170 and from the Department of Astronomy at the Ohio State University. This research has made use of the NASA/IPAC Extragalactic Database which is operated by the Jet Propulsion Laboratory, California Institute of Technology, under contract with the National Aeronautics and Space Administration.

Facilities: Hiltner (), CXO ()

\section{REFERENCES}

Abraham, R. G., Yee, H. K. C., Ellingson, E., Carlberg, R. G., \& Gravel, P. 1998, ApJS, 116, 231

Adelman-McCarthy, J. K., et al. 2008, ApJS, 175, 297

Andreon, S., de Propris, R., Puddu, E., Giordano, L., \& Quintana, H. 2008, MNRAS, 383, 102

Arnold, T., Martini, P., Mulchaey, J., \& Jeltema, T. 2009, ApJ, submitted

Assef, R. J., et al. 2008, ApJ, 676, 286

Bai, L., et al. 2007, ApJ, 664, 181

Barnes, J. E., \& Hernquist, L. 1992, ARA\&A, 30, 705

Bautz, M. W., et al. 1998, Proc. SPIE, 3444, 210

Becker, M. R., et al. 2007, ApJ, 669, 905

Berrier, J. C., Stewart, K. R., Bullock, J. S., Purcell, C. W., Barton, E. J., \& Wechsler, R. H. 2009, ApJ, 690, 1292

Bertin, E., \& Arnouts, S. 1996, A\&AS, 117, 393

Best, P. N. 2004, MNRAS, 351, 70

Best, P. N., Kauffmann, G., Heckman, T. M., \& Ivezić, Ž. 2005, MNRAS, 362, 9

Best, P. N., van Dokkum, P. G., Franx, M., \& Röttgering, H. J. A. 2002, MNRAS, 330,17

Bignamini, A., Tozzi, P., Borgani, S., Ettori, S., \& Rosati, P. 2008, A\&A, 489, 967

Bîrzan, L., Rafferty, D. A., McNamara, B. R., Wise, M. W., \& Nulsen, P. E. J. 2004, ApJ, 607, 800

Biviano, A., Katgert, P., Mazure, A., Moles, M., den Hartog, R., Perea, J., \& Focardi, P. 1997, A\&A, 321, 84
Blanton, M. R., \& Roweis, S. 2007, AJ, 133, 734

Blanton, M. R., et al. 2003, ApJ, 592, 819

Borgani, S., Girardi, M., Carlberg, R. G., Yee, H. K. C., \& Ellingson, E. 1999, ApJ, 527, 561

Branchesi, M., Gioia, I. M., Fanti, C., \& Fanti, R. 2007, A\&A, 472, 727

Branchesi, M., Gioia, I. M., Fanti, C., Fanti, R., \& Perley, R. 2006, A\&A, 446, 97

Bruzual, G., \& Charlot, S. 2003, MNRAS, 344, 1000

Butcher, H., \& Oemler, A. 1978, ApJ, 219, 18

Butcher, H., \& Oemler, A. 1984, ApJ, 285, 426

Byrd, G., \& Valtonen, M. 1990, ApJ, 350, 89

Cappelluti, N., Cappi, M., Dadina, M., Malaguti, G., Branchesi, M., D’Elia, V., \& Palumbo, G. G. C. 2005, A\&A, 430, 39

Cappi, M., et al. 2001, ApJ, 548, 624

Carlberg, R. G., Yee, H. K. C., Ellingson, E., Abraham, R., Gravel, P., Morris, S., \& Pritchet, C. J. 1996, ApJ, 462, 32

Carlstrom, J. E., Holder, G. P., \& Reese, E. D. 2002, ARA\&A, 40, 643

Chen, H.-W., et al. 2003, ApJ, 586, 745

Christlein, D., \& Zabludoff, A. I. 2003, ApJ, 591, 764

Coble, K., et al. 2007, AJ, 134, 897

Coil, A. L., et al. 2009, arXiv:0902.0363

Colless, M. 1989, MNRAS, 237, 799

Conroy, C., \& Ostriker, J. P. 2008, ApJ, 681, 151

Cooper, M. C., et al. 2008, MNRAS, 383, 1058

Cooray, A. R., Grego, L., Holzapfel, W. L., Joy, M., \& Carlstrom, J. E. 1998, AJ, 115,1388

Cowie, L. L., \& Songaila, A. 1977, Nature, 266, 501

Cowie, L. L., Songaila, A., Hu, E. M., \& Cohen, J. G. 1996, AJ, 112, 839

Czoske, O. 2004, in IAU Colloq. 195, Outskirts of Galaxy Clusters: Intense Life in the Suburbs, ed. A. Diaferio (Cambridge: Cambridge Univ. Press), 183

Dahlen, T., Mobasher, B., Somerville, R. S., Moustakas, L. A., Dickinson, M., Ferguson, H. C., \& Giavalisco, M. 2005, ApJ, 631, 126

David, L. P., Forman, W., \& Jones, C. 1999, ApJ, 519, 533

D’Elia, V., Fiore, F., Mathur, S., \& Cocchia, F. 2008, A\&A, 484, 303

Demarco, R., et al. 2005, A\&A, 432, 381

Diaferio, A., Kauffmann, G., Balogh, M. L., White, S. D. M., Schade, D., \& Ellingson, E. 2001, MNRAS, 323, 999

Dressler, A. 1980, ApJ, 236, 351

Dressler, A., \& Gunn, J. E. 1983, ApJ, 270, 7

Dressler, A., \& Gunn, J. E. 1992, ApJS, 78, 1

Dressler, A., Smail, I., Poggianti, B. M., Butcher, H., Couch, W. J., Ellis, R. S., \& Oemler, A. J. 1999, ApJS, 122, 51

Dressler, A., Thompson, I. B., \& Shectman, S. A. 1985, ApJ, 288, 481

Eastman, J., Martini, P., Sivakoff, G., Kelson, D. D., Mulchaey, J. S., \& Tran, K.-V. 2007, ApJ, 664, L9

Ebeling, H., Barrett, E., Donovan, D., Ma, C.-J., Edge, A. C., \& van Speybroeck, L. 2007, ApJ, 661, L33

Eckart, M. E., Laird, E. S., Stern, D., Mao, P. H., Helfand, D. J., \& Harrison, F. A. 2005, ApJS, 156, 35

Eckart, M. E., Stern, D., Helfand, D. J., Harrison, F. A., Mao, P. H., \& Yost, S. A. 2006, ApJS, 165,19

Elbaz, D., et al. 2007, A\&A, 468, 33

Ellingson, E., Yee, H. K. C., Abraham, R. G., Morris, S. L., \& Carlberg, R. G. 1998, ApJS, 116, 247

Ellis, S. C., \& Jones, L. R. 2004, MNRAS, 348, 165

Ettori, S., Tozzi, P., Borgani, S., \& Rosati, P. 2004, A\&A, 417, 13

Evrard, A. E., \& Henry, J. P. 1991, ApJ, 383, 95

Fang, T., et al. 2007, ApJ, 660, L27

Farouki, R., \& Shapiro, S. L. 1981, ApJ, 243, 32

Feigelson, E. D., \& Babu, G. J. 1992, ApJ, 397, 55

Finn, R. A., et al. 2005, ApJ, 630, 206

Fukugita, M., Ichikawa, T., Gunn, J. E., Doi, M., Shimasaku, K., \& Schneider, D. P. 1996, AJ, 111, 1748

Galametz, A., et al. 2009, ApJ, 694, 1309

Gallagher, S. C., Johnson, K. E., Hornschemeier, A. E., Charlton, J. C., \& Hibbard, J. E. 2008, ApJ, 673, 730

Gallazzi, A., et al. 2009, ApJ, 690, 1883

Geach, J. E., Smail, I., Moran, S. M., Treu, T., \& Ellis, R. S. 2009, ApJ, 691, 783

Geach, J. E., et al. 2006, ApJ, 649, 661

Gebhardt, K., et al. 2007, ApJ, 671, 1321

Gehrels, N. 1986, ApJ, 303, 336

Georgakakis, A., et al. 2008a, MNRAS, 385, 2049

Georgakakis, A., Gerke, B. F., Nandra, K., Laird, E. S., Coil, A. L., Cooper, M. C., \& Newman, J. A. 2008b, MNRAS, 391, 183

Ghigna, S., Moore, B., Governato, F., Lake, G., Quinn, T., \& Stadel, J. 1998, MNRAS, 300, 146 
Gilmour, R., Best, P., \& Almaini, O. 2009, MNRAS, 392, 1509

Gilmour, R., Gray, M. E., Almaini, O., Best, P., Wolf, C., Meisenheimer, K., Papovich, C., \& Bell, E. 2007, MNRAS, 380, 1467

Giovanelli, R., \& Haynes, M. P. 1985, ApJ, 292, 404

Girardi, M., \& Mezzetti, M. 2001, ApJ, 548, 79

Gisler, G. R. 1978, MNRAS, 183, 633

Goto, et al. 2005, ApJ, 621, 188

Green, P. J., et al. 2004, ApJS, 150, 43

Gunn, J. E., \& Gott, J. R. I. 1972, ApJ, 176, 1

Harrison, F. A., Eckart, M. E., Mao, P. H., Helfand, D. J., \& Stern, D. 2003, ApJ, 596, 944

Hasinger, G., Miyaji, T., \& Schmidt, M. 2005, A\&A, 441, 417

Heinis, S., et al. 2007, ApJS, 173, 503

Hopkins, P. F., Hernquist, L., Cox, T. J., Di Matteo, T., Robertson, B., \& Springel, V. 2006, ApJS, 163, 1

Houghton, R. C. W., Magorrian, J., Sarzi, M., Thatte, N., Davies, R. L., \& Krajnović, D. 2006, MNRAS, 367, 2

Ilbert, O., et al. 2005, A\&A, 439, 863

Jee, M. J., White, R. L., Ford, H. C., Illingworth, G. D., Blakeslee, J. P., Holden, B., \& Mei, S. 2006, ApJ, 642, 720

Jeltema, T. E., Canizares, C. R., Bautz, M. W., \& Buote, D. A. 2005, ApJ, 624, 606

Jeltema, T. E., Mulchaey, J. S., Lubin, L. M., \& Fassnacht, C. D. 2007, ApJ, 658,865

Johnson, O., Best, P. N., \& Almaini, O. 2003, MNRAS, 343, 924

Johnson, O., et al. 2006, MNRAS, 371, 1777

Kaiser, N. 1991, ApJ, 383, 104

Kauffmann, G., et al. 2004, MNRAS, 353, 713

Kelson, D. D., van Dokkum, P. G., Franx, M., Illingworth, G. D., \& Fabricant, D. 1997, ApJ, 478, L13

Kewley, L. J., Geller, M. J., \& Jansen, R. A. 2004, AJ, 127, 2002

Kim, D.-W., et al. 2004a, ApJS, 150, 19

Kim, D.-W., et al. 2004b, ApJ, 600, 59

Kim, M., et al. 2007, ApJS, 169, 401

Kocevski, D. D., Lubin, L. M., Gal, R., Lemaux, B. C., Fassnacht, C. D., \& Squires, G. K. 2009, ApJ, 690, 295

Koester, B. P., et al. 2007, ApJ, 660, 239

Kosowsky, A. 2003, New Astronomy Rev., 47, 939

Kravtsov, A. V., Berlind, A. A., Wechsler, R. H., Klypin, A. A., Gottlöber, S., Allgood, B., \& Primack, J. R. 2004, ApJ, 609, 35

Larson, R. B., Tinsley, B. M., \& Caldwell, C. N. 1980, ApJ, 237, 692

Lehmer, B. D., et al. 2007, ApJ, 657, 681

Lehmer, B. D., et al. 2009, ApJ, 691, 687

Lin, Y.-T., \& Mohr, J. J. 2007, ApJS, 170, 71

Lubin, L. M., Mulchaey, J. S., \& Postman, M. 2004, ApJ, 601, L9

Marcillac, D., Rigby, J. R., Rieke, G. H., \& Kelly, D. M. 2007, ApJ, 654, 825

Margon, B., Spinrad, H., \& Downes, R. A. 1983, Nature, 301, 221

Martel, A. R., Menanteau, F., Tozzi, P., Ford, H. C., \& Infante, L. 2007, ApJS, 168,19

Martini, P. 2004, in IAU Symp. 222, The Interplay among Black Holes, Stars and ISM in Galactic Nuclei, ed. T. Storchi-Bergmann, L. C. Ho, \& H. R. Schmitt (Cambridge: Cambridge Univ. Press), 235

Martini, P., Kelson, D. D., Kim, E., Mulchaey, J. S., \& Athey, A. A. 2006, ApJ, 644, 116

Martini, P., Kelson, D. D., Mulchaey, J. S., \& Trager, S. C. 2002, ApJ, 576, L109

Martini, P., Mulchaey, J. S., \& Kelson, D. D. 2007, ApJ, 664, 761

McNamara, B. R., \& Nulsen, P. E. J. 2007, ARA\&A, 45, 117

Mei, S., et al. 2006, ApJ, 644, 759
Mei, S., et al. 2009, ApJ, 690, 42

Merritt, D. 1983, ApJ, 264, 24

Metcalfe, L., Fadda, D., \& Biviano, A. 2005, Space Sci. Rev., 119, 425

Miller, C. J., Nichol, R. C., Gómez, P. L., Hopkins, A. M., \& Bernardi, M. 2003, ApJ, 597, 142

Miller, N. A., Owen, F. N., Hill, J. M., Keel, W. C., Ledlow, M. J., \& Oegerle, W. R. 2004, ApJ, 613, 841

Mink, D. J. 2002, in ASP Conf. Ser. 281, Astronomical Data Analysis Software and Systems XI, ed. D. A. Bohlender, D. Durand, \& T. H. Handley (San Francisco, CA: ASP), 169

Moore, B., Katz, N., Lake, G., Dressler, A., \& Oemler, A., Jr. 1996, Nature, 379,613

Mushotzky, R. F., \& Scharf, C. A. 1997, ApJ, 482, L13

Muzzin, A., Yee, H. K. C., Hall, P. B., \& Lin, H. 2007, ApJ, 663, 150

Örndahl, E., Rönnback, J., \& van Groningen, E. 2003, A\&A, 404, 883

Osterbrock, D. E. 1960, ApJ, 132, 325

Ota, N., \& Mitsuda, K. 2004, A\&A, 428, 757

Poggianti, B. M., et al. 2006, ApJ, 642, 188

Poggianti, B. M., et al. 2008, ApJ, 684, 888

Popesso, P., \& Biviano, A. 2006, A\&A, 460, L23

Postman, M., Lubin, L. M., \& Oke, J. B. 2001, AJ, 122, 1125

Rauzy, S., Adami, C., \& Mazure, A. 1998, A\&A, 337, 31

Richstone, D. O. 1976, ApJ, 204, 642

Ruderman, J. T., \& Ebeling, H. 2005, ApJ, 623, L81

Ruhl, J., et al. 2004, in SPIE Conf. Ser. 5498, 11

Saintonge, A., Tran, K.-V. H., \& Holden, B. P. 2008, ApJ, 685, L113

Silverman, J. D., et al. 2005, ApJ, 618, 123

Silverman, J. D., et al. 2008, ApJ, 679, 118

Silverman, J. D., et al. 2009a, ApJ, 695, 171

Silverman, J. D., et al. 2009b, ApJ, 696, 396

Sivakoff, G. R., Martini, P., Zabludoff, A. I., Kelson, D. D., \& Mulchaey, J. S. 2008, ApJ, 682, 803

Stanford, S. A., Holden, B., Rosati, P., Tozzi, P., Borgani, S., Eisenhardt, P. R., \& Spinrad, H. 2001, ApJ, 552, 504

Stern, D., Holden, B., Stanford, S. A., \& Spinrad, H. 2003, AJ, 125, 2759

Sun, M., \& Murray, S. S. 2002, ApJ, 577, 139

Sunyaev, R. A., \& Zeldovich, Y. B. 1970, Ap\&SS, 7, 3

Tran, K.-V. H., Franx, M., Illingworth, G. D., van Dokkum, P., Kelson, D. D., Blakeslee, J. P., \& Postman, M. 2007, ApJ, 661, 750

Tran, K.-V. H., van Dokkum, P., Illingworth, G. D., Kelson, D., Gonzalez, A., \& Franx, M. 2005, ApJ, 619, 134

Treu, T., et al. 2003, ApJ, 591, 53

Ueda, Y., Akiyama, M., Ohta, K., \& Miyaji, T. 2003, ApJ, 598, 886

van Breukelen, C., et al. 2009, MNRAS, 395, 11

van Dokkum, P. G. 2001, PASP, 113, 1420

van Dokkum, P. G., \& Franx, M. 1996, MNRAS, 281, 985

van Dokkum, P. G., Franx, M., Fabricant, D., Illingworth, G. D., \& Kelson, D. D. 2000, ApJ, 541, 95

van Dokkum, P. G., \& van der Marel, R. P. 2007, ApJ, 655, 30

Vikhlinin, A., VanSpeybroeck, L., Markevitch, M., Forman, W. R., \& Grego, L. 2002, ApJ, 578, L107

Wang, Q. D., Owen, F., \& Ledlow, M. 2004, ApJ, 611, 821

Wolf, C., Meisenheimer, K., Rix, H.-W., Borch, A., Dye, S., \& Kleinheinrich, M. 2003, A\&A, 401, 73

Xue, Y.-J., \& Wu, X.-P. 2000, ApJ, 538, 65

Yencho, B., Barger, A. J., Trouille, L., \& Winter, L. M. 2009, ApJ, 698, 380

Zentner, A. R., Berlind, A. A., Bullock, J. S., Kravtsov, A. V., \& Wechsler, R. H. 2005, ApJ, 624, 505 